\title{
Network effects and spatial autoregression in mode choice models: Three essays in urban transportation economics
}

Frank Goetzke

West Virginia University

Follow this and additional works at: https://researchrepository.wvu.edu/etd

\section{Recommended Citation}

Goetzke, Frank, "Network effects and spatial autoregression in mode choice models: Three essays in urban transportation economics" (2006). Graduate Theses, Dissertations, and Problem Reports. 2497. https://researchrepository.wvu.edu/etd/2497

This Dissertation is protected by copyright and/or related rights. It has been brought to you by the The Research Repository @ WVU with permission from the rights-holder(s). You are free to use this Dissertation in any way that is permitted by the copyright and related rights legislation that applies to your use. For other uses you must obtain permission from the rights-holder(s) directly, unless additional rights are indicated by a Creative Commons license in the record and/ or on the work itself. This Dissertation has been accepted for inclusion in WVU Graduate Theses, Dissertations, and Problem Reports collection by an authorized administrator of The Research Repository @ WVU.

For more information, please contact researchrepository@mail.wvu.edu. 
Network Effects and Spatial Autoregression in Mode Choice Models:

Three Essays in Urban Transportation Economics

\author{
Frank Goetzke \\ Dissertation submitted to the \\ College of Business and Economics \\ at the West Virginia University \\ in partial fulfillment of the requirements for the degree of
}

Doctor of Philosophy

in

Economics

David R. Martinelli, Ph.D., Chair

Brian J. Cushing, Ph.D.

Stratford M. Douglas, Ph.D.

Randall W. Jackson, Ph.D.

Jerald J. Fletcher, Ph.D.

Santiago M. Pinto, Ph. D.

Division of Economics and Finance

Morgantown, West Virginia

2006

Keywords: Transportation Economics, Network Externalities, Neighborhood Effects, Mode Choice Model, Spatial Econometrics

(C) Copyright 2006, Frank Goetzke 


\section{Abstract \\ Network Effects and Spatial Autoregression in Mode Choice Models: Three Essays in Urban Transportation Economics}

\section{Frank Goetzke}

Network analysis in transportation economics has traditionally focused on congestion as a negative externality stemming from supply-side capacity constraints. In my first paper paper, an analytical mode choice model is developed to examine the demand-side network effects. The assumption behind the approach is that, because of social network effects, the utility of people taking the mode increases with its mode share. It is found that social network effects change the modal aggregate demand curve for the mode to an inverted u-shape. This result has far-reaching policy consequences, since multiple equilibria become a possibility, causing positive externalities and path-dependency.

Transportation planners have always been aware of positive network effects in public transit use, which can be attributed to the fact that people choose transit, because other people already take it. In my second essay, I employ a spatially autoregressive mode choice mode to econometrically test for the existence of social network effects. It is found that the coefficient estimate for transit use network effects is positive and significantly different from zero. Furthermore, if social network effects are not included, it can be shown that an omitted variable bias is introduced into the model, which can lead to a systematic error in travel forecasts.

The third essay explains municipal differences in bicycle mode share with social network effects. Using data from the nation-wide travel behaviour survey, Mobility in Germany 2002, a binary logistic regression model was developed to identify in how much a city-specific "biking culture” has an impact on the city's bike modal split. To avoid endogeneity of the biking culture variable, a social network effects instrument was developed. It was found that not only bicycle infrastructure, but also social network effects change municipal bike mode share. Further results were that work/educational and leisure trips depend less on social network effects than other trip purposes. The outcome of this research has significant policy implications, such as, that transportation planners can target biking culture in a city as a mean to improve bike mode share. 
To my beloved wife Rebecca Potter, who opened a whole New World for me.

And to my lovely daughters Evelyn and Tahlia, who opened yet another new world for me.

All three are reminding me constantly, that life is about people (and academia about their humanity). 


\section{Acknowledgements}

I am grateful to Dr. David Martinelli, who for his unconditional support not only as his research assistant but also his friendship during my study for the Ph.D. His strong interest in transportation issues and his interdisciplinary approach to the field was and still is an inspiration for me. He helped me not only to ask the right questions, but also prepared me for my future job by giving me a good understanding about the academic profession and research. I am deeply indebted to him for the patience and time he spent with me despite all his obligations as the Department chair, which made my work easier to accomplish and my academic achievements possible.

I would also like to express gratitude to Dr. Brian Cushing, who introduced me to and gave me a deep appreciation for analyzing urban problems with economic tools. During the dissertation process, he always enabled me to not only see the single trees but also the forest as a whole. Without his thoughtful advice, it would have been not as easy to finish the project. Needless to say, his clear and insightful comments on my work were of tremendous help for me.

I want to thank Dr. Santiago Pinto with whom I had long discussions about my ideas and how to formulate them more rigorously, and Dr. Stratford Douglas who supported me on all the econometrical questions I had, especially with the technical problems that arose in conducting my empirical research.

Both Dr. Jerald Fletcher and Dr. Randall Jackson lent their valuable support and their comments on the finished dissertation, which strengthened the work as a whole.

I am glad that I had the opportunity to take short training courses with both Dr. Luc Anselin and Dr. James LeSage. Both scholars introduced me to spatial econometrics, 
but beyond this, they opened a door for me by giving me a more spatial understanding of the world. It helped me in writing my dissertation and, I am convinced, that it also will create great opportunities in my future academic career.

The last dissertation essay would have been not possible without the support of Dr. Tilmann Rave from the IFO Institute for Economic Research at the University of Munich in Germany, where I stayed as a visiting researcher for part of the academic year 2004/05. I owe him my thanks.

Both my fellow student colleague Justin Ross, as well as my friend Thomas Kornmann helped me a great deal to finish my dissertation. Justin Ross did text editing, and Thomas Kornmann supported my data collection efforts by making a lot of calls to German municipal governments.

Finally, I want to acknowledge Dorothea Hass of WalkBoston and Douglass Lee of the Volpe Center, as well as the staff of my former employers, Central Transportation Staff and Cambridge Systematics, especially Steve Falbel, Lauri Hussey, Sam Lawton and Kevin Tierney for how they shaped my thinking about transportation and supported me in my career as a transportation professional.

I am also grateful for the assistance from the New York Metropolitan Transportation Council and the DLR Clearinghouse for Transportation Data (Clearingstelle Vekehr am Deutschen Zentrum für Luft- und Raumfahrt) in providing me with the data for the second and third chapters of my dissertation, without which these two studies would have been impossible.

Last, but not least, I am deeply indebted to my family, namely my wife Rebecca Potter, who supported me morally, assisted in the proof-reading and editing, as well as 
my two daughters Evelyn and Tahlia, who both had to get used to me walking off in order to write my dissertation, after they had gotten used to me as a stay-home father.

I thank all of these people for their assistance and support in making this a better—and most importantly, a completed—work. All errors are of course my own. 


\section{Table of Contents}

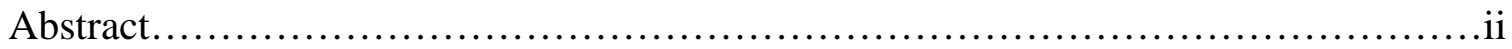

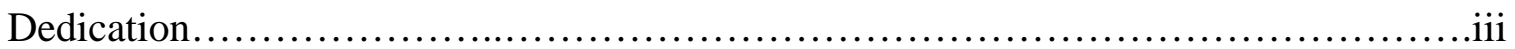

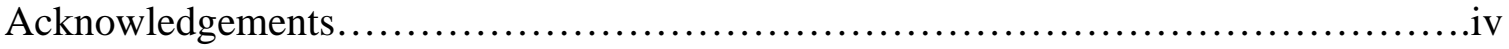

Table of Contents................................................................vii

List of Tables..................................................................

List of Figures............................................................... viii

Chapter $1 \quad$ Introduction.....................................................

Chapter 2 Mode Choice and Social Networks: The Economics

of Walking, Bicycling and Public Transit Use..........................7

Chapter 3 Social Network Effects and Public Transit Use: Evidence from

a Spatially Autoregressive Mode Choice Model..............................46

Chapter 4 Bicycle Use in Germany: Explaining Differences between

Municipalities with Social Network Effects..............................76

Chapter $5 \quad$ Conclusion........................................................ 111 


\section{List of Tables}

Table 3.1: $\quad$ Estimation results for the New York City Mode Choice Model.

Table 4.1: $\quad$ Summary statistics for included model variables.

Table 4.2: $\quad$ Municipal characteristics used in the model.

Table 4.3: Coefficients for the correlations of the network proxies with total bike model share and the two different infrastructure variables.

Table 4.4(a): Binary logistic regression results for bicycle trips using "Bike Lanes per Capita” as the infrastructure measure. The network effect proxy is the bike mode share of the excluded trip purpose.

Table 4.4(b): Binary logistic regression results for bicycle trips using "Bike Infrastructure Score” as the infrastructure measure. The network effect proxy is the bike mode share of the excluded trip purpose.

Table 4.5(a): Marginal effects for regressions (1) trough (4).

Table 4.5(b): Marginal effects for regressions (5) trough (8).

Table 4.6: Regression results for city-specific dummies as the dependent variable.

\section{List of Figures}

Figure 2.1: $\quad$ Positive network effects for an individual person in their mode choice.

Figure 2.2: Linear distribution of the preference for walking with others $m_{x}$ over the group of all $n$ potential pedestrians.

Figure 2.3: $\quad$ Phase diagram for the u-shaped aggregate walking demand curve with network effects. 
Figure 2.4: Aggregate walking demand curve for a combination of per person different preference for walking with others as well as for different WTP for walking independent of others.

Figure 2.5: A realistic example of all combination of preference to walk with others, $M$, and minimum WTP for walking, $b$ (explanation see text).

Figure 2.6: Welfare analysis for walking demand with positive network effects, assuming a constant $b_{x}$.

Figure 2.7: The collapse of transit use from $s_{1}$ to $s_{2}$ after automobile cost drops from $c_{1}$ to $c_{2}$ (example for the United Stated between 1945 and 1975).

Figure 2.8: The u-shaped aggregate demand curve for walking pivots around the origin as soon the marginal congestion cost is included and decreases the willingness to pay.

Figure 2.9: With congestion on the alternative mode, the aggregate demand curve pivots around the point of a transit share 1 . This can result into a traditionally downward sloped demand curve.

Figure 3.1: Positive network effects for an individual person in their mode choice decision.

Figure 3.2: Geo-coded location of all survey households in New York City.

Figure 3.3: In the presence of network effects transit utility increases with higher transit mode share. However, if network effects are not included into the model, transit utility is forced to be constant, which leads to an omitted variable bias. Therefore, transit ridership will be systematically underestimated in the suburbs $\left(\mathrm{v}_{1}<\mathrm{v}_{2}\right)$, and overestimated in the central city $\left(\mathrm{v}_{1}>\mathrm{v}_{2}\right)$. 
Chapter 1

Introduction 
During the last decade it became more acceptable to analyze sociological topics with economic tools. In the field of social interactions, economists began looking at herd behavior, cultural conventions, foreign language learning, gift giving and fashion. The concepts of social network or spillover effects are the centerpiece of all these works.

What are social network effects? Very simply defined, social network effects exist if people prefer to do what other people already do. One reason for this behavior is that what other people do is viewed as an information signal. A person could believe that if someone else does a certain activity, this someone else may have additional information about this activity. For example, if a restaurant is crowded, people think is must be a good place to eat. Fashion or peer pressure are different interpretations of network effects.

If a person prefers to walk, bicycle or use public transit as long he or she sees other people using the same mode, then the concept of social network effects also applies to transportation mode choice. In the transportation context, social network effects can arise due to an externality, where the central issue becomes a coordination problem. For example, while all members of a group together would benefit by riding transit, if nobody is willing to take the first step in using it the pay-off goes uncollected. 
Until recently nobody had applied these ideas towards transportation mode choice modeling. Transportation planners were always aware of social network effects. They knew that pedestrians like busy sidewalks, or people prefer biking when they see other bicyclists. Even more, they understood that if a transit system loses enough riders, it may easily collapse. The lack of these demand-side network research in the area of transportation is especially astonishing because supply-side network externalities, such as congestions, were one of the major issues transportation science dealt with during the last half century.

In the last few years, mainly two groups of authors, Antonio Páez and Darren Scott at McMaster University in Canada, as well as Joan Walker at Boston University and Elenna Dugundji at the University of Amsterdam in the Netherlands, have worked on very similar questions. All of them developed approaches to integrate demand-side network effects into their empirical travel behavior models.

However, my dissertation differs from the above research in three significant ways: First, I have developed a theoretical framework to evaluate social network effects as an externality problem and discuss their consequences. I found that social network effects in transportation mode choice modeling can lead to multiple equilibria of this transportation mode, and, 
therefore, this transportation mode share would be path-dependent. The policy implications are far-reaching, since modal split, such as walking in American cities, results from potentially irreversible historical events.

Second, I model empirically the social network effects as a spatial autoregressive process, rather than using a simple zonal mode share mean. My approach of a spatially weighted modal split moving average is not only more realistic, it also takes care of the endogeneity problem of the network variable. Spatial data, however, is not always available. In these cases I find an instrument for the social network effects.

Third, my research has a strong focus on policy matters. My major contribution lies in making the transportation planning community aware of the consequences of social network effects. Since ignoring network externalities may lead to ill-informed decision making, I conclude all three essays with a policy discussion that focuses on the relevance of my findings.

The dissertation comprises three essays, presented in Chapters 2, 3, and 4. In the next chapter, I lay out a theoretical framework for mode choice decision making that includes social network effects. Starting off with an analytical model, I find that social network effects may lead to multiple mode share equilibria causing path-dependency. This means that a city's actual modal split (of e.g. pedestrians, bicyclists or transit riders) may follow unique 
historical events, and may be irreversible. A discussion of the welfare implications shows that only the equilibrium with the highest level of the mode share is welfare maximizing.

After generalizing the one-mode model to a two-mode model, I extend the basic model to include congestion either on the mode exposed to social network effects, or the alternate mode. I find that, for congestion on the network-exposed mode, a congestion charge is not necessarily welfare maximizing. Furthermore, if the alternate mode (e.g. automobile) is congested, the externality of the network-exposed mode (e.g. transit) could be easier to overcome. The results shed new light on the post-World War II transit ridership collapse in the United States.

Based on the New York City household travel dataset, I then develop in Chapter 3 a mode choice model to evaluate the presence of social network effects in public transit use. Social network effects are modeled as a spatially autoregressive transit mode share process. The regression coefficient estimates for the social network effects variable were positive and significantly different from zero at the 5-percent level. I also show that, if the mode choice model does not account for social network effects, the estimates of the regression coefficients exhibit an omitted variable bias. The consequences discussed in the conclusion of the chapter are the following: Transit ridership is 
overestimated for trips going from the suburbs into the CBD, while it is underestimated for trips within the central city. This systematic bias in the model can become the cause for poor decision making by transportation planners.

Finally, in Chapter 4 I analyze following the concept of social network effects, municipal bicycle mode share differences in German cities. The new idea is that a person's decision to ride the bike depends also on the biking city’s culture in which he or she lives. Using a national travel behavior dataset, I build a binary logistic regression model to model bicycle mode choice as a function of personal, trip and city-specific variables, plus social network effects.

I employed a city-level instrumental variable, based on the bike mode share of some excluded records (by trip purpose), and found that the regression coefficient estimates were positive in all cases, as well as significantly different from zero at the 5-percent level. The impact of two different bicycle infrastructure proxies was found to be positive, as well. With this empirical evidence of bicycle mode choice decision making depending on social network effects, transportation planners may focus not only on infrastructure improvements, but also on strengthening the city’s biking culture. 
In Chapter 5, I finish up with a brief conclusion, summarizing all the results and giving an outlook onto further research in the field of network effects in transportation mode choice modeling. 


\section{Chapter 2}

\section{Mode Choice and Social Networks:}

The Economics of Walking, Bicycling and Public Transit Use 


\section{INTRODUCTION}

Ever since the publication of Studies in the Economics of Transportation (Beckmann, McGuire and Winston, 1956), network analysis in transportation economics has centered on congestion as a negative externality. The foundation of this research is the limited capacity of the physical transportation network. ${ }^{1}$ This paper builds upon and extends this approach by also examining the positive network externalities stemming from the social networks of people choosing certain transportation modes, namely walking, bicycling and public transit. While traditional analysis is based on network effects in the supply of transportation, my focus is on network effects in transportation demand. The idea behind this new concept is simple: For some transportation modes, such as walking, bicycling, or transit use, the willingness-to-pay for people considering this mode increases with the number of other people already taking the same mode. This seems counterintuitive in the context of capacity constraints for transportation networks, since traditionally an additional person on the same network link is thought as imposing a congestion externality on everyone else already using the link. While it is possible to include capacity constraints and congestion in my argument, I did so only in the very last section of this paper, because for

\footnotetext{
${ }^{1}$ For a survey on network analysis in transportation and beyond see Nagurney (2003).
} 
walking, bicycling and public transit the maximum capacity is typically very high and rarely reached - a large city, such as New York, may be an exception.

Why do people prefer to walk, bike, or use public transit together with others? The motivations can be grouped into three categories: The first category is a utility gain through the network effects coming from some kind of complementarity, since people are not alone, can meet other people, communicate with them, and feel safer. The second category is based on conformity and can be described as avoiding a utility loss by not following others because of the social norm, peer pressure and/or fashion. And in the last category, the utility improvement stems from internalizing an information externality, because people using a certain transportation mode (i.e. public transit) send a signal to everyone else that this is a feasible mode (public transit is reliable and, therefore, works) ${ }^{2}$.

Including these social networks in mode choice decision-making is just an additional aspect of current network analysis in transportation. I am not claiming that these effects are more or less important than congestion. However, this minor extension of accounting for positive network effects, even if not given much weight, alters the result rather significantly.

\footnotetext{
${ }^{2}$ This is similar to choosing a restaurant because its popularity is interpreted as a sign of quality.
} 
Traditional economic reasoning assumes a downward sloping aggregate transportation demand curve; however, I will show that, if network effects exist in the mode choice decision, the demand curve changes to an inverted u-shape. This fact leads to the possibility of multiple equilibria, which has far-reaching consequences. First, a critical mass of users is necessary to make a transportation mode feasible or a transportation facility utilized. Second, this critical mass requirement may lead to local clusters of a specific transportation mode, which means mode choice decisions become spatially autoregressive. And third, potential multiple equilibria would make the actual mode share outcome path-dependent and, thus, a result of unique historical events. All three consequences are observable in real mode choices decisions, such as walking, bicycling, and transit use, and have important policy implications to be discussed in the conclusion.

While these insights are commonly known in the economics of network industries (Economides, 1996; Rohlfs, 1974; Shy, 2001), they have not been appropriately applied in the context of transportation networks. Yevdokimov (2001 and 2002) incorporated demand-side network effects in his general equilibrium model of optimal highway investment, but he did not theoretically justify its use or further develop the concept. Goodwin et. al. (2005) mention demand-side network effects for transit users in their microeconomics textbook 
without further developing the argument. Also, there is an emerging literature that finds econometric evidence of demand-side network effects in mode choice decision making (Dungundij and Walker, 2006; Goetzke, 2006), or simulates network effects in travel behavior (Páez and Scott, 2005; 2006). This literature, however, does not discuss the phenomena using an analytical model derived from economic theory as presented in this paper. Of course, there is also a body of economics literature concerning herd behavior (Banerjee, 1992), as well as social interaction (Akerlof, 1997), elaborating on externalities and multiple equilibria, but they do not explicitly deal with transportation mode choice decision-making.

In the next section, I will introduce the basic mode choice model, consisting of one transportation mode. Then, I will relax some of the restrictions for the one-mode model and develop a general form of the basic model, which will be used for the social welfare discussion in the following section. In the next section, the model will be extended to two modes, before finally congestion is added to the model. I finish with a conclusion.

\section{THE BASIC MODEL}

Loosely following the pioneering paper by Rohlfs (1974), where he analyzes the demand structure of telecommunication services in the presence of 
network externalities, I will present a static partial equilibrium model, where the marginal cost of using the transportation mode is exogenously determined. The basic model includes positive network externalities, but not congestion (a negative network externality) and allows a person to either use a certain transportation mode for the trip or not do the trip at all. The assumption is that all people are homogeneous in every aspect, except for their preference for choosing the mode together with others and they all face the same marginal cost using this mode.

Before delving too deeply into the theoretical aggregate model, I want to first discuss graphically the impact of a positive network effect on the demand curve of an individual person choosing to walk. As seen in Figure 2.1(a), the downward-sloping walking demand curve shifts up with a higher pedestrian mode share, which means that at the same level of walking consumption, the willingness-to-pay (WTP) for walking increases. Figure 2.1(b) exhibits the resulting upward sloping curve of WTP for one walking trip with respect to pedestrian mode share, which is a good starting point for introducing the basic model.

\section{Figure 2.1 about here}


Consider now a group of $n$ people choosing to undertake a nonessential trip where the only possible transportation mode is walking ${ }^{3}$. Assuming a mode preference for walking with others of $m_{x} \geq 0^{4}$, the utility $U_{x}$ for a specific person $x$ taking this walking trip is defined as follows:

$$
U_{x}= \begin{cases}m_{x} s^{e}-p & \text { if the person walks and } \\ 0 & \text { if the person does not walk. }\end{cases}
$$

The first term in Equation (1), which is the product of the personal mode preference for walking with others, $m_{x}$, and the expected pedestrian mode share $s^{e} \geq 0$, represents the network effect. The second part of the equation, $p \geq 0$, denotes the full cost of walking. Since a low preference to use the modes with others, $m_{x}$, will result in a value closer to zero, it can be easily seen that this leads to the cost of walking becoming more dominant when compared to the network effect. However, as the mode preference grows stronger, the walking cost term will lose its relative importance.

Now I can analyze a specific person $x$ ' who is indifferent about walking or not walking. This would be the case as long as:

$$
m_{x^{\prime}} s^{e}-p=0
$$

\footnotetext{
${ }^{3}$ The assumption of a single transportation mode, such as walking, simplifies the analysis for better understanding. A later extension of the model will add more realism by allowing an alternative mode.

${ }^{4}$ If $m_{x}<0$, then the person dislikes walking with others, which would be a congestion case.
} 


$$
p=m_{x}, s^{e}
$$

Equation (2) resembles exactly the network effect for an individual person as seen in Figure 2.1(b). The larger the preference to use the mode with others, $m_{x}$, the steeper the slope of the WTP curve in the graph and the more pronounced the network effect. A walking preference of zero would cause a horizontal WTP curve and a walking preference of infinity, a vertical WTP curve.

In order to derive the aggregate demand curve, I first need to determine the distribution for the preference to use the mode with others, $m_{x}$, over all $n$ people. The simplest case would be a uniform distribution for $m_{x}$, which is defined as a linear equation, where person $x$ is mapped to the preference for using the mode with others, $m_{x}$, as follows:

$$
m_{x}=M-{ }^{M} / n x
$$

$M$ is the maximum value for $m_{x}$ and $n$ is the number of people potentially walking. In this set-up, the first person on the left of the $x$-axis has the highest preference to walk with others, while the last person on the right side of the $x$ axis has the lowest preference to walk with others (Figure 2.2).

\section{Figure 2.2 about here}


In order to derive the aggregate demand curve, I have to use Equation (3) for a specific person $x$ ' in Equation (2):

$$
\begin{aligned}
& p=m_{x}, s^{e} \\
& p=\left(M-M / n x^{\prime}\right) s^{e}
\end{aligned}
$$

In Equation (4), I know that while person $x$ ' is indifferent towards walking, all people indexed as $x<x$ ' become definitely pedestrians, since their preference for walking with others is higher than for person $x$ '. Therefore, assuming perfect foresight, I can simply say that the expected pedestrian mode share is equal to the number of actual pedestrians:

$$
s^{e}=1 / n x
$$

The inverse aggregate demand function for walking can now be derived by substituting Equation (5) into Equation (4). I also want to normalize $x$ ' to the pedestrian share $s=x / n$ :

$$
\begin{aligned}
& p=\left(M-M / n x^{\prime}\right) \frac{1}{n} x^{\prime} \\
& p=\left(s-s^{2}\right) M
\end{aligned}
$$

The aggregate demand curve, as seen in Figure 2.3, has an inverted ushape, is upward sloping at low levels of walking and becomes downward sloping after reaching the maximum WTP of $1 / 4 M$ at a pedestrian mode share of $1 / 2$. This means that as long as the mode share is less than $1 / 2$ the WTP for 
walking increases with every additional pedestrian, which is a result of the positive network externality. Assuming constant returns to scale, a horizontal marginal cost curve can be added to Figure 2.3. The level of the marginal cost curve $(M C)$ will be determined by travel time, the quality of the walking facilities (the higher the quality, the lower the cost), and other factors of convenience, such as weather and/or aesthetics.

\section{Figure 2.3 about here}

As long the marginal cost is above the maximum WTP $(M C>1 / 4 M)$, such as in the example of $p_{1}$, the mode share will be zero $\left(s_{l}\right)$, as seen in point $A$ in Figure 2.3. However, if the marginal cost drops below $1 / 4 M$, $p_{2}$ will intersect three times with the demand curve, leading to two stable outcomes ( $B$ and $D$ ), as well as to one unstable outcome $(C)$ according to the phase diagram. With outcome $B$, there are still no pedestrians, but if outcome $D$ is achieved, the mode share for walking will be $s_{2}$. The existence of two stable equilibria can result in two similar walking facilities with the same marginal cost, while facing the same aggregate demand curve can potentially have two different levels of utilization, one at zero and the other significantly above. 


\section{THE GENERAL FORM OF THE BASIC MODEL}

The basic model above has quite a few restrictions that I want to relax now. The major restriction is the uniformly distributed network preference. The question is whether or not the inverted u-shape of the aggregate demand curve holds also for skewed mode preference distributions. To discuss the cases of a larger number of people having a high or low preference for walking with others, I can formulate the following general equation for the network preference distribution:

$$
m_{x}=\left[1-\left({ }^{x} / n\right)^{a}\right] M \text {, with } a \geq 0
$$

The basic model discussed in the section above exists for $a=1$. If $a>1$, however, then there are more people with high preferences to walk with others, while as long as $0<a<1$, there are more people with low preferences to walk with others. Substituting Equation (7) into Equation (2) and normalizing for pedestrian share using Equation (5), I can find the following aggregate demand function:

$$
p=\left(s-s^{a+1}\right) M
$$

The characteristic of an inverted u-shape does not change with this addition, but the peak moves to the right of the center (pedestrian share equals $1 / 2$ ) for $a$ $>1$, and to the left for $0<a<1$. If $a=0$, which identifies the case where 
everybody has the same preference to walk with others at $M$, the inverted ushaped aggregate demand curve flattens to a horizontal line at the level of $M$.

So far, I have assumed that people are not willing to pay for walking independently of the positive network effect. If I relax this assumption, another term, $b_{x} \geq 0$, can be added to Equation (1), which could be seen as a parameter of a personal preference for walking. If aggregated, the functional form of $b(x)$ depends on the distribution of $b_{x}$ over all potential pedestrians, which, in this context, I do not need to restrict with any assumptions. Therefore, the general form of the aggregate demand curve is now:

$$
p=\left(s-s^{a+1}\right) M+b(x)
$$

What the minimum WTP for walking independent of others, $b_{x}$, does, is shifting the individual WTP curve up by its value with respect to pedestrian mode share. Now it is possible to derive the traditionally downward sloping aggregate demand curve by assuming that all people have the same preference of walking with others of 0 , but they also have a decreasing WTP, which is independent of others as well as uniformly distributed between $B$ and 0 . This would result in the aggregate demand curve starting on the left at the value of $B$ for a pedestrian share of 0 and ending on the right at the value of 0 for a pedestrian share of 1 . By combining different preferences for walking with 
others, $m_{x}$, and different WTP for walking independent of others, $b_{x}$, I can derive the aggregate demand curve seen in Figure 2.4.

\section{Figure 2.4 about here}

Figure 2.4 shows the individual demand curves with respect to the pedestrian mode share based on their individual combinations of network and personal walking preferences: The first person on the far left has a personal walking preference of $B_{3}$, but does not care about walking with others (no network effects). Moving from left to right, the next person has a personal walking preference of $\mathrm{B}_{2}$ and a network preference equal to the slope of her individual demand curve. The following person has the same personal walking preference as the person before, but, again, her network preference is equal to 0 . The next person (in the middle) has a personal preference of 0 , but now the network preference is the same as the one of the second person (same slope of the demand curve). The next person has again the same personal walking preference of 0 , but a lower network preference than the previous person (lower slope). Finally, the two following people have both the same network preferences (parallel demand curves), however, the very last person, on the far right, has a personal walking preference of 0 , while the person before has a 
personal walking preference of $\mathrm{B}_{1}$. If all the individual demand curves are combined, an aggregate demand curve can be derived, such as exhibited in Figure 2.4. With increasing pedestrian mode share, which means moving in the graph from left to right, the highest WTP value is selected for each level of pedestrian mode share, to derive the aggregate demand curve.

Finally, I have to relax the assumption of $m_{x}$ being a real number. As long as I have people who always walk independent of others, I have to allow $m_{x}$ to become infinity. The result would be a vertical, inelastic aggregate demand curve. In Figure 2.5, I have combined all possible cases (discussed above) into a more realistic aggregate demand curve. On the far left I start with a share of $s_{1}$ people having a preference value of walking with others of infinity, then, still on the left, I continue with a group having high values of $m_{x}$, where the change of the minimum WTP, $b_{x}$, is more dominant than the network effect (until $s_{2}$ ), followed by a group to the right, where the network effect dominates the change of $b\left(s_{3}\right)$, and finish on the far right with a group of people having a preference to walk with others of 0 and a decreasing value of $b_{x}$

\section{Figure 2.5 about here}




\section{SOCIAL WELFARE DISCUSSION}

In order to be able to decide which of the two stable equilibria is welfare maximizing, I have to evaluate Equation (4) and (8) assuming that the expected pedestrian mode share is the actual pedestrian mode share $s^{*}$. Since all pedestrians with a larger value for their preference to use the mode with others (on the left of $s^{*}$ ) also have a higher WTP then the actual cost at $s^{*}$, there will be a consumer surplus. In order to derive the consumer surplus, I need to find the aggregate demand curve at the fixed level of $s^{e}=s^{*}$. To simplify the exercise, I assume that $b(x)$ is equal to 0 :

$$
\begin{aligned}
& p=\left(M-M s^{a}\right) s^{e} \\
& p=M s^{*}-\left(M s^{*}\right) s^{a}
\end{aligned}
$$

As seen in Figure 2.5, the aggregate demand curve at the actual level of pedestrian share $s^{*}$ is classically downward sloping and intersects with the inverted u-shaped aggregate demand curve at the point $s^{*}=s_{1}$. In the graph I assume that the walking preference parameter $b_{x}$ is the same for everyone at a level of $B$.

Using Equation (10), the consumer surplus for the stable right-hand, non-zero equilibrium outcome at $s^{*}$ can be estimated as follows:

$$
C S={ }_{0} s^{*}\left[M s^{*}-\left(M s^{*}\right) s^{a}\right] d s
$$




$$
\begin{aligned}
& =1 / 2 M S^{*}-(1 / a+1) M\left(s^{*}\right)^{a+2}+k \\
& =M s^{2}\left(1 / 2-1 / a+1 *^{* a}\right)+k \geq 0 \text { for } 1 / 2 \leq s^{*} \leq 1 \text { and } a \geq 1
\end{aligned}
$$

The constant of integration is $k$. Assuming that there is no producer surplus, the right-hand stable equilibrium with a pedestrian mode share of $s_{1} \geq 1 / 2$ (point $B$ ) must be the welfare maximizing outcome $(C S \geq 0)$, because the consumer surplus will be always greater then for a pedestrian share of 0 (point $\mathrm{A}$ ), where the consumer surplus is just zero.

\section{Figure 2.6 about here}

However, the actual mode share outcome is path-dependent. Therefore the welfare maximizing result is not guaranteed. This can typically be seen in new pedestrian infrastructure investments, which indeed decrease the marginal cost of walking without necessarily increasing its usage. In order to take advantage of the positive network effects, a subsidy may be needed, at least in theory, ideally paid to the people with the highest preference to walk with others. Then the zero-equilibrium will move towards the welfare maximizing

equilibrium. In practice, this subsidy could be an informational advertisement campaign or consist of other kinds of promotions for the new infrastructure, such as group walks, which first convinces people with a large value for their 
preference to walk with others to use the new infrastructure. Then other pedestrians should follow as soon as the critical mass is passed. ${ }^{5}$ In the context of transit, a monthly pass may also make the people with the highest network preference willing to start using the bus, with the result that others may follow.

In summary, it can be said, since the inverted u-shaped aggregate demand curve exhibits positive network effects causing changing WTP curves for each pedestrian mode share level, that the area underneath the demand curve is not equal to the consumer surplus.

\section{THE TWO-MODE MODEL}

Most transportation mode choice decisions involve at least two alternatives, such as public transit and the automobile. This extended model will therefore account for two modes, one with positive network externalities (public transit) and one without any network externalities. The change is conceptually easy to include into the general form of the basic model. All previous assumptions remain, except now everyone has to undertake the trip with one of both transportation modes. Therefore, nobody can choose to not take the trip at all:

\footnotetext{
${ }^{5}$ To refer back to the previously mentioned restaurant example, the first few customers receive a free meal or discount, is a typical approach use by restaurants to take advantage of the positive network effects.
} 


$$
U_{x}= \begin{cases}m_{x} s^{e}-p+b_{x} & \text { if the person uses transit and } \\ -c+d_{x} & \text { if the person takes the car }\end{cases}
$$

In Equation (12), $p \geq 0$ is the full cost for using transit, which is the fare, but could also include the travel time as well as the mode's disamenities. The full cost of driving a car (operation, parking and travel time) is represented by $c$, while $d_{x} \geq 0$ is the individual WTP for driving the car, again a parameter of personal preference for driving ${ }^{6}$. After manipulating Equation (12), not assuming a functional form for the distribution of $d_{x}$, it can be shown that the aggregate demand curve just shifts upwards by the amount of the automobile cost $[c-d(x)]$, while all previously discussed characteristics remain, including the potential for multiple equilibria:

$$
p=\left(s-s^{a+1}\right) M+b(s)+[c-d(x)]
$$

As soon as the cost of the alternative mode to transit, automobile cost, $c$, decreases, the inverted u-shaped aggregate demand curve shifts down as expected with the result of a lower transit share. This means, because of the inverted u-shape characteristics of the aggregate demand curve that transit could lose its critical mass of users. Transit mode share would collapse from $s_{1}$ to $s_{2}$ with the decrease of the automobile operating cost from $d_{1}$ to $d_{2}$ (see

\footnotetext{
${ }^{6}$ In the context of the individual WTP curve $p=m_{x} s^{e}+b_{x}+c-d_{x}$, the term $\left(b_{x}-d_{x}\right)$ becomes the personal preference for walking relative to driving.
} 
Figure 2.7). Therefore, positive network effects may provide a good explanation for what has happened to the public transit mode share between the end of World War II, 1945, and the first Oil Crisis, 1975. During this time transit ridership declined in the United States at a very rapid rate as a result of increased access to cars and lower automobile operation costs (APTA, 1995; Clair, 1981). When using my explaination, however, I do not have to assume a change in the preferences towards either public transit or the automobile, represented in the WTP $b(s)$ and $d(s)$, respectively. Nor do I even have to assume a lower quality of transit service, which would result in an upward shift of the marginal cost curve, $p$, as is common in the conspiracy theories surrounding this issue (Slater, 1997). Both an additional change of individual preferences away from public transportation and towards the car, as well as a lower transit service quality, would further accelerate the described process.

\section{Figure 2.7 about here}

\section{ADDING CONGESTION TO THE MODEL}

At last, I want to add congestion to the models discussed above. There are two possible cases to consider: The first case is where marginal social cost for the transportation mode exhibiting positive network effects is greater than 
its marginal private cost. The question in this context is if a congestion toll equal to the externality is efficient as is the case without the network effects. In the second case, I want to discuss consequences in the aggregate demand curve when the alternative mode is congested.

Using the basic model in its general form, I now assume that the walking facility can get congested, which means that with a higher pedestrian share it is slower to walk because of crowding. Assuming that the congestion cost is equal for everyone, I can subtract a congestion term $f(s)$ from Equation (8), which is a positive function of the pedestrian share. After again manipulating the equation as previously done, the following aggregate demand function can be found:

$$
p=\left(s-s^{a+1}\right) M+b_{x}-f(s)
$$

In equation (14) the WTP for walking falls at the rate of increasing congestion cost, with the result that the inverted u-shaped aggregate demand curve pivots around the origin down to the left and gets compressed to scale. As seen in Figure 2.8, this leads to a flatter inverted u-shape of the aggregate demand curve, as well as one which intersects with the $\mathrm{x}$-axis before the pedestrian share reaches the value of one. Since the supply curve representing the cost of walking did stay the same, the change could lead to a zero mode share equilibrium because the WTP for walking is at all points less than the cost. 


\section{Figure 2.8 about here}

So far the analysis has only taken into account that an additional pedestrian faces a higher cost of walking. However, to complete the picture, this pedestrian also imposes a congestion externality on all already existing pedestrians. The traditional approach for internalizing this congestion cost on others is to charge a toll in the amount of this congestion externality. But with positive network effects, there may be cases where a congestion toll may not be welfare maximizing. To see that, consider again Equation (14). A congestion toll of $t(s)$ would further decrease the willingness to pay (the inverted u-shape becomes even more flat) with the possible consequence of moving a stable right-hand equilibrium with a positive pedestrian share to a zero equilibrium. If that happens, it can be stated that as long as the consumer surplus of the equilibrium without the congestion toll minus the total external cost from the congestion is greater than zero, not charging a congestion toll is welfare maximizing, since the alternative is a consumer surplus of zero.

It is possible that a congestion toll may not be welfare maximizing even if the congestion toll does not lead to a zero mode share equilibrium, because the aggregate demand curve at a fixed level of pedestrian share is changing 
with the actual pedestrian share (see again Figure 2.6). Therefore, it can happen that the consumer surplus of the equilibrium without the congestion toll minus the total cost from the congestion is still greater than the consumer surplus of the equilibrium with congestion cost. This result stems from the fact that the positive network effect is an external benefit, counteracting the effect coming from the congestion externality.

In summary, it can be said that as long as the externality caused by the positive network effect cannot be internalized, as it would be typically the case in the real, second-best world, a congestion toll can turn out to be economically inefficient. Since the finding also holds for the two-mode model, it is especially important to stress in the context of congested transit systems that instituting a congestion-based fare system could lead to suboptimal outcomes.

Now I want to evaluate congestion cost occurring in the alternative mode of a two-mode model. Using transit as the mode with positive network effects and the automobile as the alternative transportation mode, a negative congestion term, $g(1-s)$, which is a positive function of how many do not use transit $(1-s)$, needs to be added to the automobile cost, so that Equation (13) changes as follows:

$$
\begin{aligned}
p & =\left(s-s^{a+1}\right) M+[b(s)-d(s)]+[c+g(1-s)] \\
& =\left(s-s^{a+1}\right) M+[b(s)-d(s)]+[c-g(s)]
\end{aligned}
$$


Interpreting Equation (15), in Figure 2.9 it can be seen that the congestion term pivots the aggregate transit demand curve up to the right around the point where the transit share is 1 , and, therefore, increases the WTP for transit at the amount of the automobile congestion cost. The result is that the inverted u-shaped aggregate transit demand curve is lifted up on the left, essentially decreasing the positive network characteristics. In cities with high congestion costs, this again can lead to a purely downwardly sloped aggregate demand curve for transit, which coincides with the empirically observed facts of large, congested cities with a functioning transit system. This also explains why automobile congestion prevents the collapse of transit use as previously described.

Furthermore, if a congestion toll would be charged for the automobile mode, equal to the external cost an additional driver imposes on all the other already existing drivers, then this congestion toll would further lift the aggregate transit demand curve up on the left, and would cause an increased WTP for transit at low transit share levels. Thus, a congestion toll would not only, increase transit ridership as expected, but may prevent transit systems close to collapse from doing so. 


\section{POLICY CONCLUSION}

Transportation planners always knew about the existence of network effects for the walking, bicycling and transit modes. However, nobody formalized the theory as I have done, so that it becomes possible to derive clear and unambiguous policy recommendations.

Since the aggregate demand curve derived for transportation modes exhibiting positive network effects has an u-shape which can lead to multiple equilibria, there are two major conclusions to draw from this research: On the one hand, transportation modes such as walking, biking and/or transit require a critical mass because of the u-shaped demand curve. On the other hand, the actual mode share outcome is path-dependent and historically determined because of the existence of multiple equilibria.

Despite the historic uniqueness of actual local transportation mode share equilibrium, I also expect that the actual mode share outcomes are locally clustered, because of the spill-over characteristics of networks, and therefore spatially correlated. The result would be pockets of very high walking or transit use in a certain city, while other areas of the same city have extremely low shares of these transportation modes. This is exactly what can be observed in large cities, such as New York, where it can be found that in parts of Manhattan the pedestrian mode share for walking trips is almost half, while 
other neighborhoods have a transit mode share well above 50\%. As econometrically shown in Goetzke (2006), this phenomenon cannot exclusively be explained by travel time, but also needs to be informed by the theory of positive network effects.

Goetzke (2006) further finds that if mode choice decisions are spatially autoregressive, a systematic bias in traffic forecasting will be introduced into the mode choice model, severely impacting the foundation of policy decision making in the context of new infrastructure investment.

Additional policy-relevant findings are as follows: It is not enough to just build new and better infrastructure with the intent of raising the mode share for this transportation mode. As long as the current mode share is at the zero equilibrium it takes more to convince people to use new and better facilities, which can be seen with new pedestrian, biking and/or transit facilities in areas with virtually no pedestrians, bikers or transit users.

Therefore, it is crucial for cities with a still functioning transit system to prevent transit ridership to drop below the critical mass. Improving automobile traffic may eventually lead to an irreversible collapse of transit with the consequence that drivers are also worse off. In this context it is important to mention that in a city the more people who depend on transit as their sole mode 
of transportation, the more likely it is, that a person who owns an automobile will use transit, as well, because of network effects.

Finally, promoting walking, biking and/or transit use and improving information about these transportation modes may make it possible that the mode share moves from the zero equilibrium to a right-hand equilibrium, since marketing and advertisement campaigns function as a subsidy, essentially internalizing the external network benefit.

\section{REFERENCES}

APTA (1995) Transit Fact Book. Washington, DC: American Public Transportation Association.

Akerlof, G. A. (1997) Social Distance and Social Decisions, Econometrica, 65, pp. 1005-1027.

Banerjee. A. V. (1992) A Simple Model of Herd Behavior, Quarterly Journal of Economics, 107, pp. 787-817.

Beckmann, M., C. B. McGuire and C. B. Winston (1956) Studies in the Economics of Transportation. New Haven, CT: Yale University Press. 
Clair, D. J. St. (1981) The Motorization and Decline of Urban Public Transit 1935-1950, Journal of Economic History, 41, pp. 579-600.

Economides, N. (1996) The Economics of Networks, International Journal of Industrial Organization, 14, pp. 673-699.

Dugundij, E. R. and J. L. Walker (2006) Discrete Choice with Social and Spatial Interdependencies. An Empirical Example Using Mixed GEV Models with Field and “Panel” Effects, Transportation Research Record, forthcoming.

Goetzke, F. (2006) Social Network Effects in Public Transit Use: Evidence from a Spatially Autoregressive Mode Choice Model, Urban Studies, forthcoming.

Goodwin, N., J. A. Nelson, F. Ackerman and T. Weisskopf (2005) Microeconomics in Context. Boston, MA: Houghton Mifflin Company.

Nagurney, A. (2003) Influence of Beckman, McGuire, and Winsten’s Studies in the Economics of Transportation on Innovations in Modeling, Methodological Developments, and Applications. Philadelphia, PA: 50th Annual North American Meetings of the Regional Science Association International. 
Rohlfs, J. (1974) A Theory of Interdependent Demand for a Communication Service, The Bell Journal of Economics and Management Science, 5, pp. 16-37.

Páez A. and D. M. Scott (2005) A Discrete Choice Approach to Modeling Social Influence on Individual Decision Making. Seattle, WA: 51st Annual North American Meetings of the Regional Science Association International (revised version of the presentation).

Páez A. and D. M. Scott (2006) Social Influence on Travel Behavior: A Simulation Example of the Decision to Telecommute, Environment and Planning $A$, forthcoming.

Shy, O. (2001) Economics of Network Industries. Cambridge, UK and New York, NY: Cambridge University Press.

Slater, C. (1997) General Motors and the Demise of Streetcars, Transportation Quarterly, 51, pp. 45-66.

Yevdokimov, Y. (2001) The Economics of Transportation Networks. Fredericton, Canada: 30th Atlantic Canada Economic Association Conference. 
Yevdokimov, Y. (2002) Modelling Transportation as a Network Industry.

Aix-En-Provence, France: 8th International Computing in Economics and Finance Conference. 
Figure 2.1: Positive network effects for an individual person in their mode choice decision.

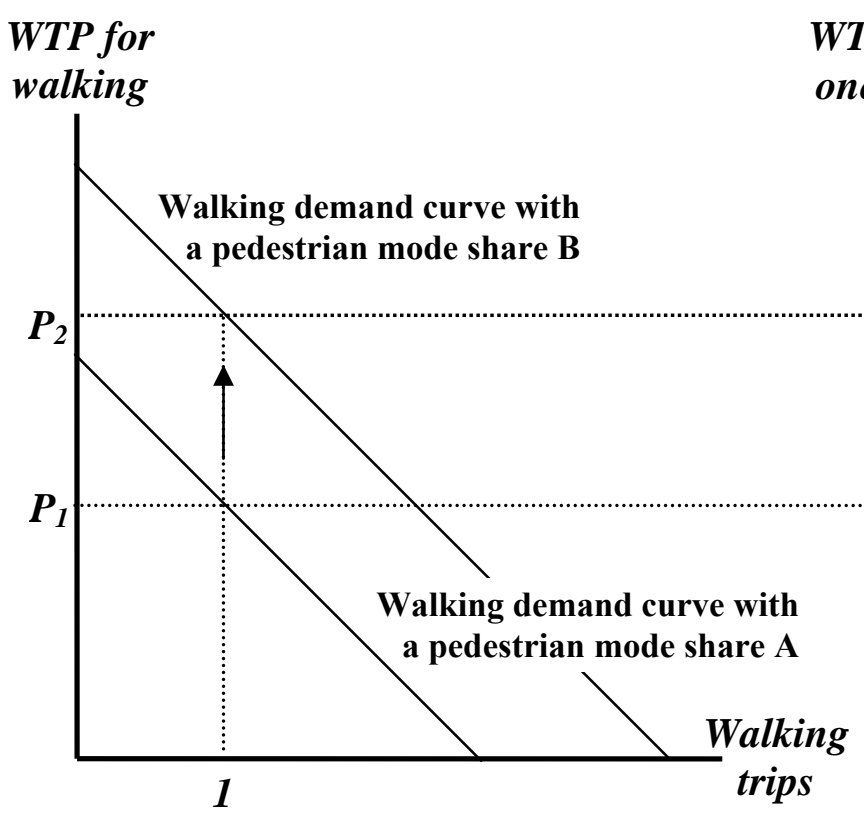

(a) With higher mode share increases the WTP for walking and the demand curve shifts up (positive network effect).

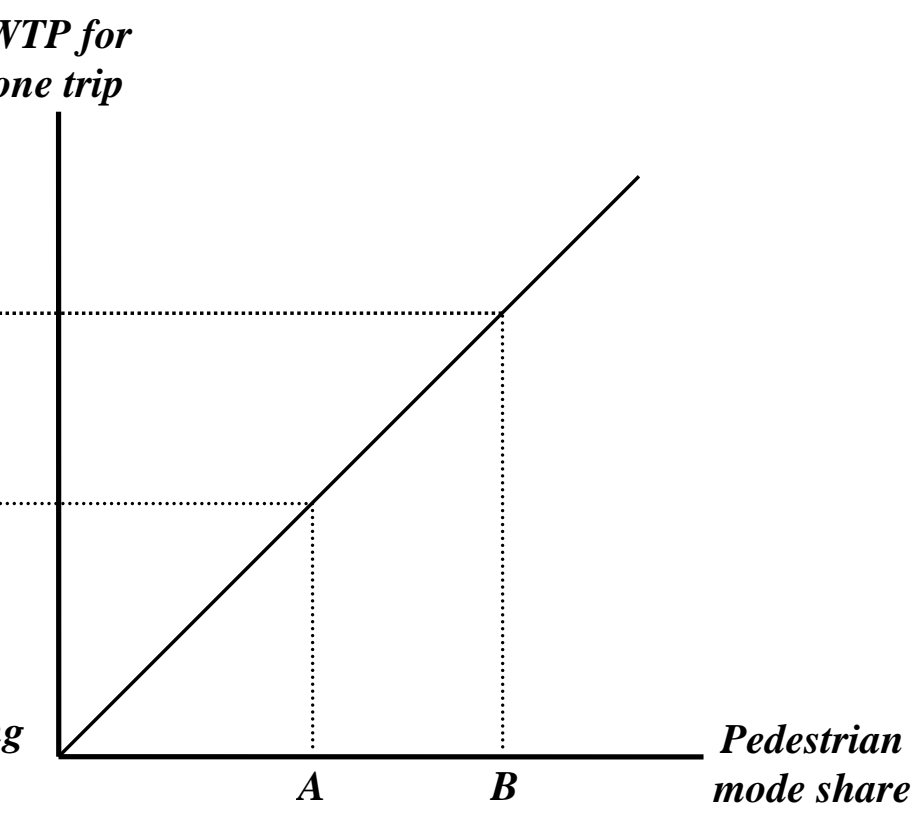

(b) Because of the network effect, the curve of WTP for one walking trip w.r.t. to pedestrian mode share is an upward sloping curve. 
Figure 2.2: Linear distribution of the preference for walking with others $m_{x}$ over the group of all $n$ potential pedestrians.

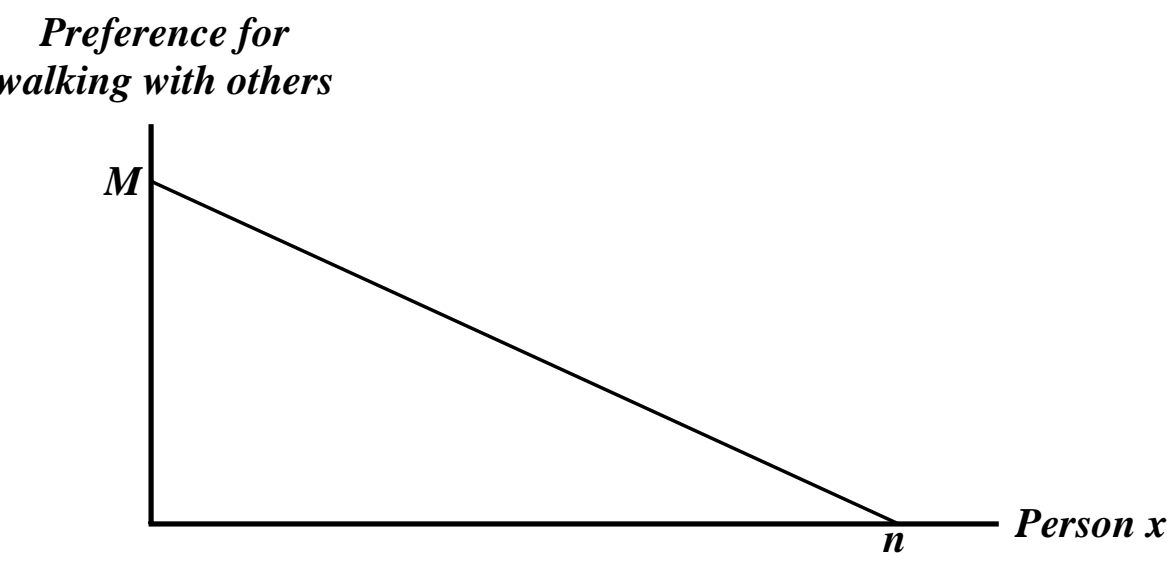


Figure 2.3: Phase diagram for the u-shaped aggregate walking demand curve with network effects.

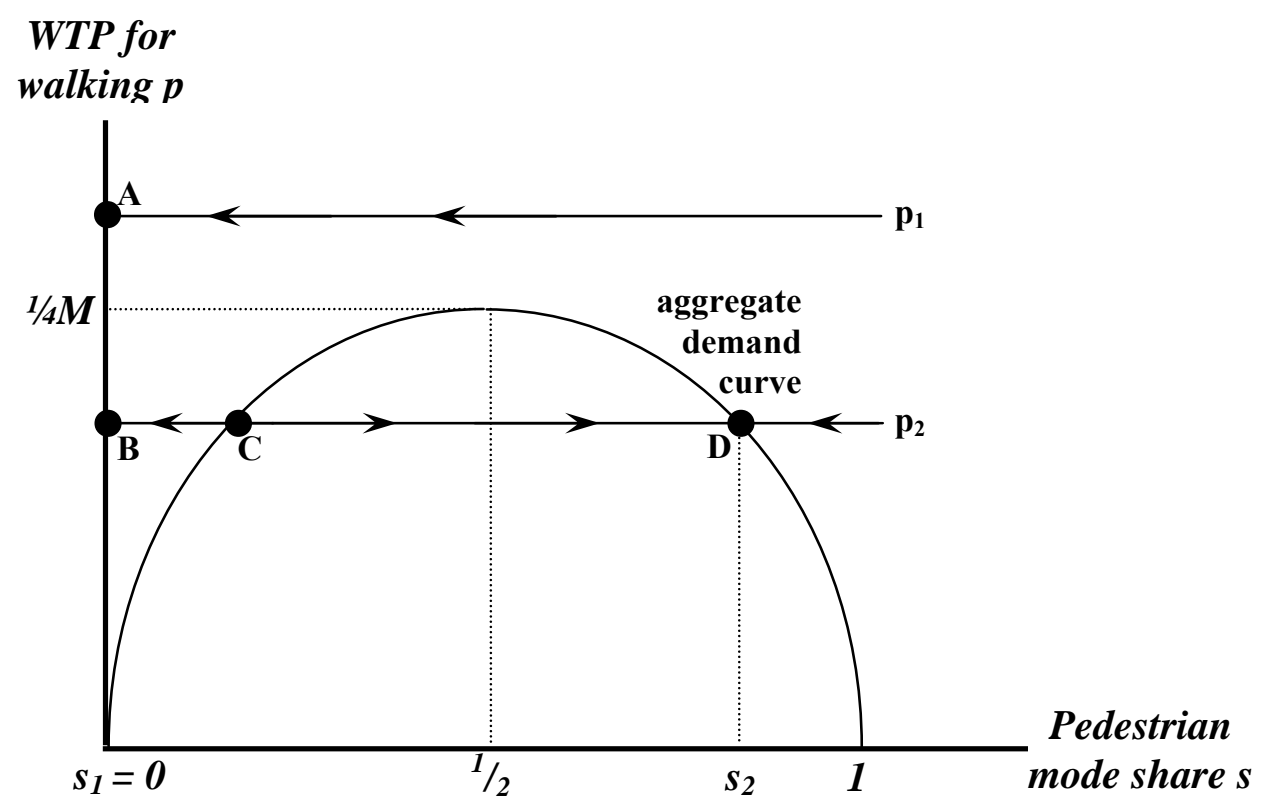


Figure 2.4: Aggregate walking demand curve for a combination of per person different preference for walking with others as well as for different WTP for walking independent of others.

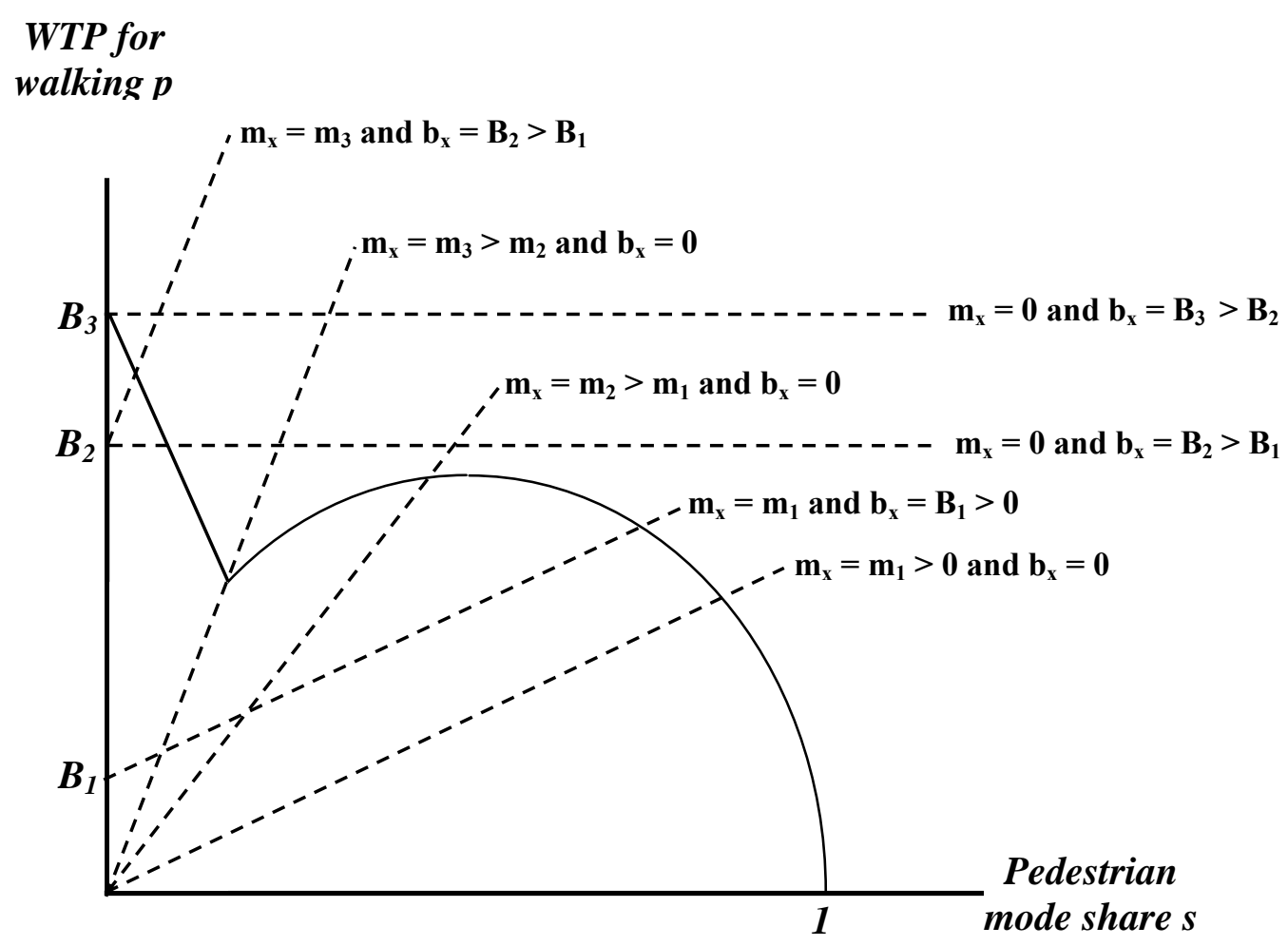


Figure 2.5: A realistic example of all combination of preference to walk with others, $M$, and minimum WTP for walking, $b$ (explanation see text).

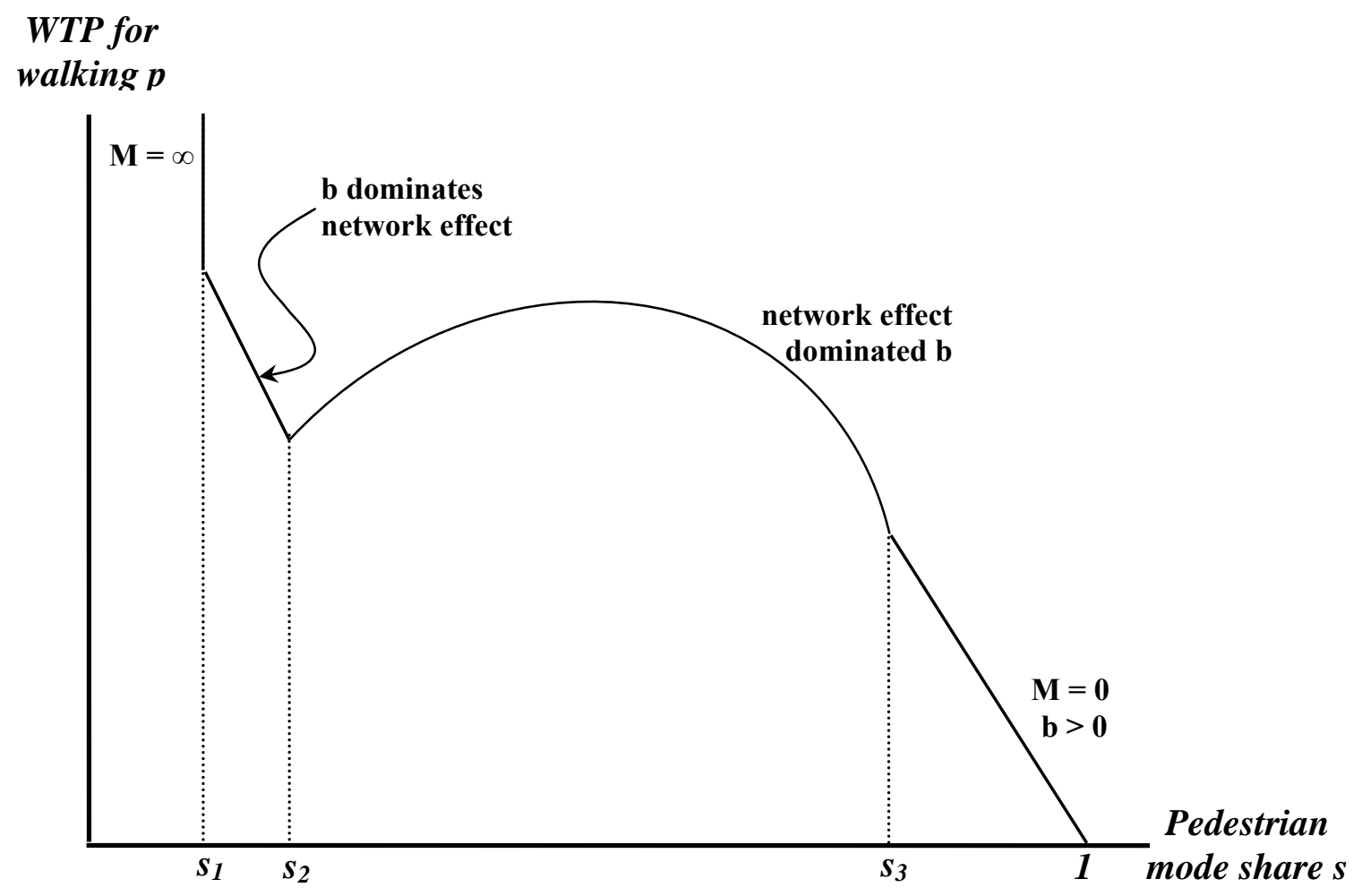


Figure 2.6: Welfare analysis for walking demand with positive network effects, assuming a constant $b_{x}$.

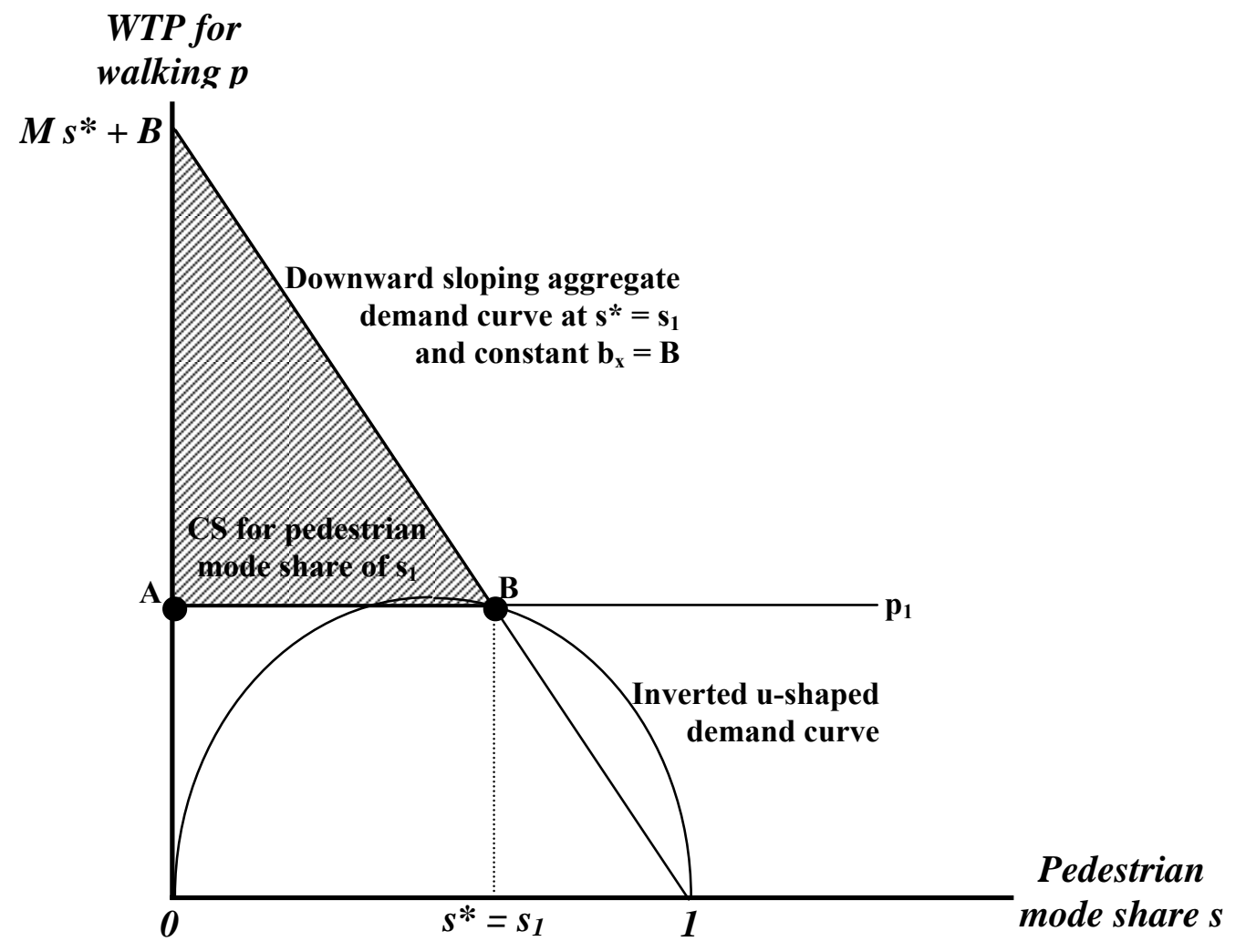


Figure 2.7: The collapse of transit use from $s_{1}$ to $s_{2}$ after automobile cost drops from $c_{1}$ to $c_{2}$ (example for the United Stated between 1945 and 1975).

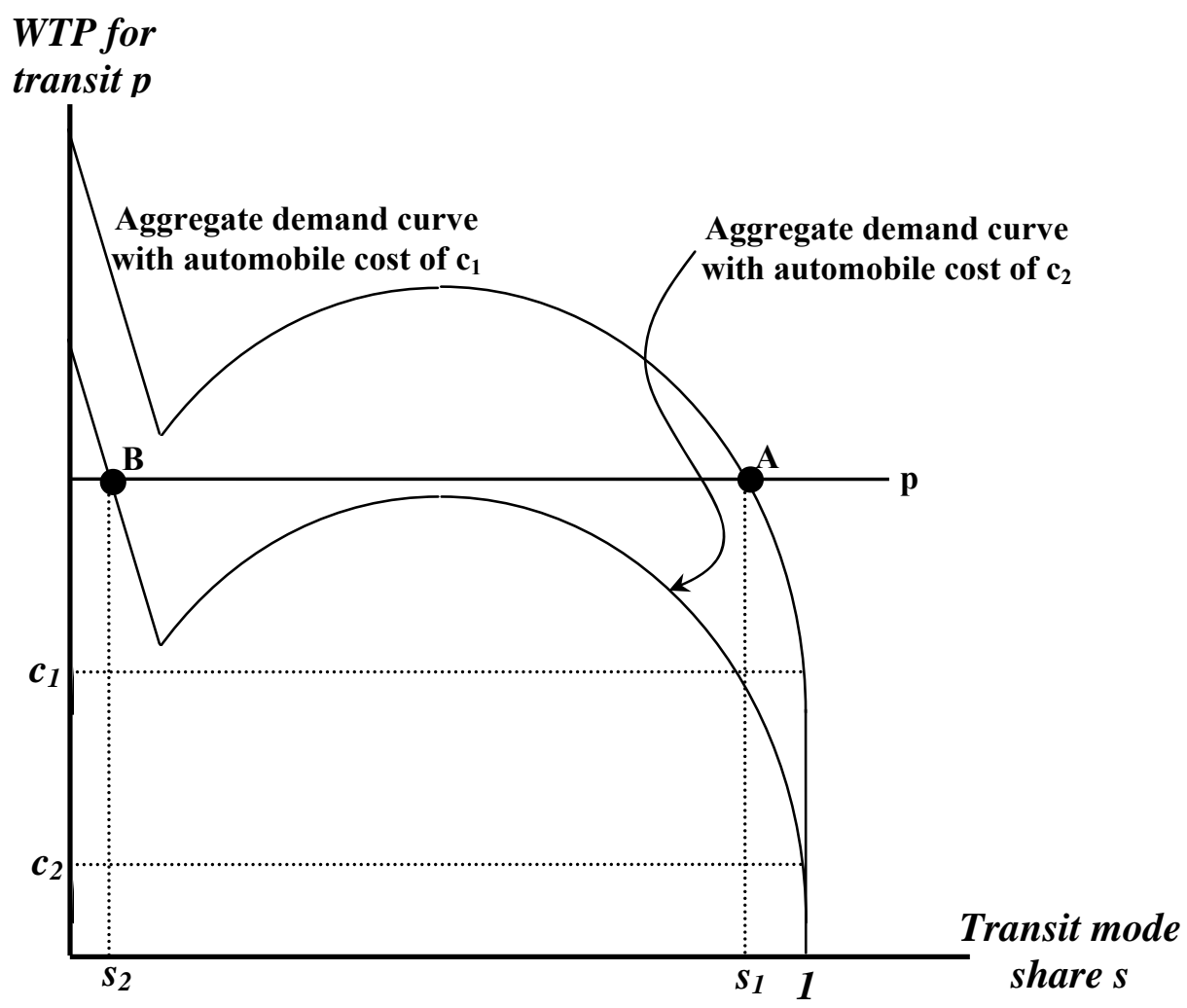


Figure 2.8: The u-shaped aggregate demand curve for walking pivots around the origin as soon the marginal congestion cost is included and decreases the willingness to pay.

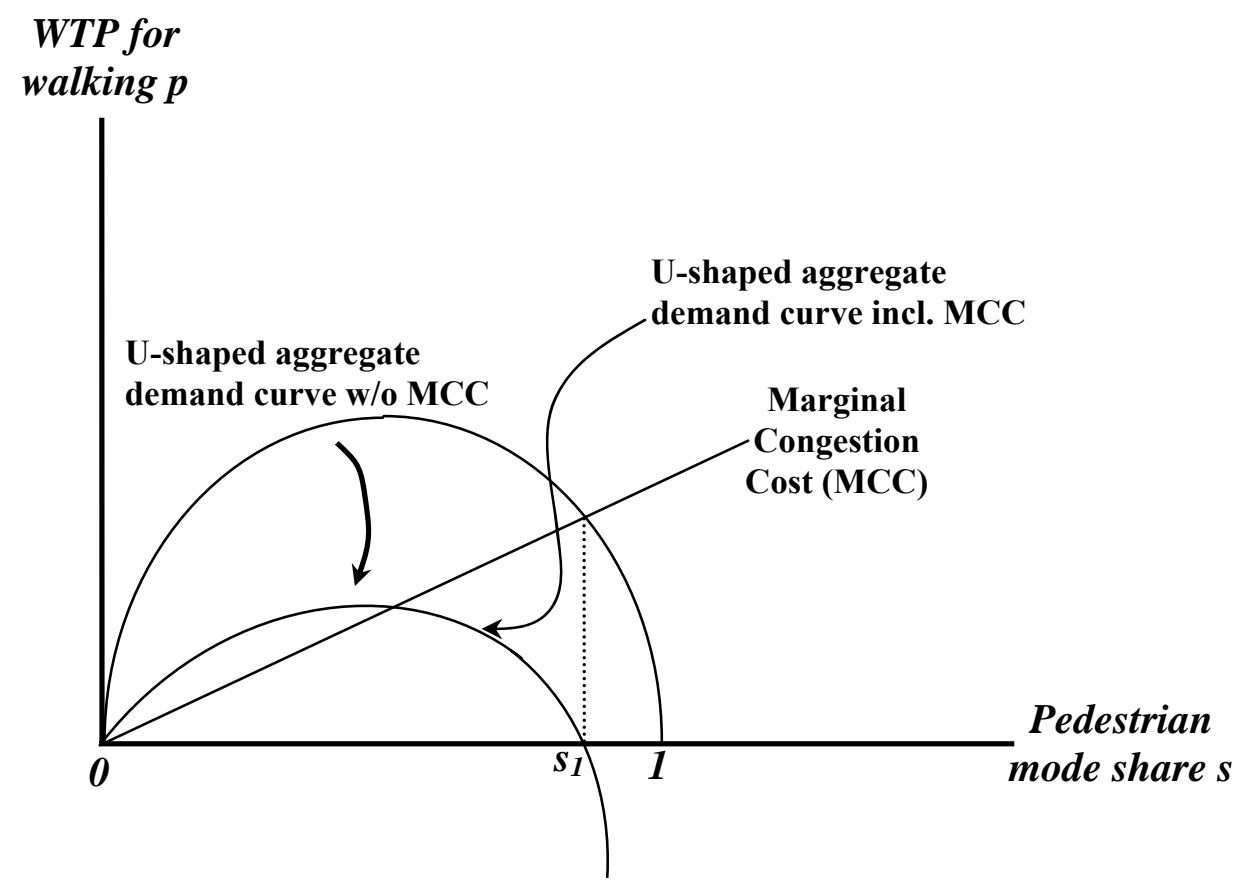


Figure 2.9: With congestion on the alternative mode, the aggregate demand curve pivots around the point of a transit share 1 . This can result into a traditionally downward sloped demand curve.

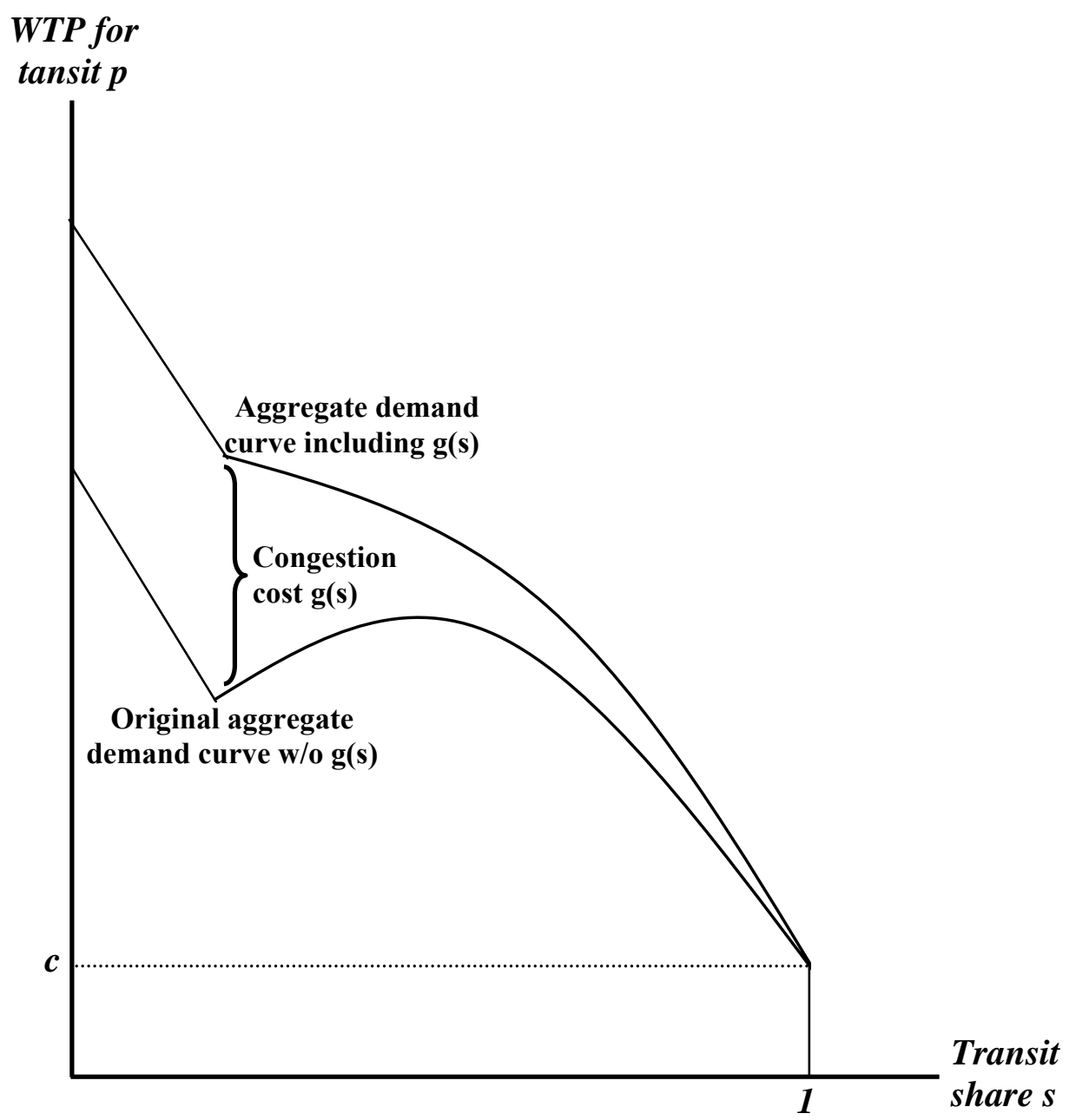




\section{Chapter 3}

Social Network Effects in Public Transit Use:

Evidence from a Spatially Autoregressive Mode Choice Model 


\section{INTRODUCTION}

In this paper, I empirically test for positive network effects in transit use by applying a spatial autoregressive logit mode choice model with 1997/98 work trip data from New York City. Positive network effects exist when people prefer to use transit together with other people as a result of social spill-over. Although these network preferences should differ for each person, because of statistical restrictions in the model, I cannot get individual network preferences. However, I will be able to econometrically derive a measure of their aggregate network preference.

There is increasing interest in analyzing spatial dependencies and network effects in travel behavior. LeSage and Polasek (2005) examine commodity flow matrices by extending a gravity model, a tool widely used in the field of transportation, to include spatial autocorrelation. Using a Monte Carlo simulation, Páez and Scott (2005; 2006), investigate in two forthcoming articles the impact of social networks in discrete choice models.

It is not entirely new to include network effects into empirical choice models. Brock and Durlauf have been researching methods to account for social interaction and neighborhood effects in both binary, as well as multinomial choice models (Brock and Durlauf, 2001; 2002). However, they do not explicitly include a spatial dimension to the discussion of the problem. 
On the other hand, very few spatial discrete choice models have been estimated because of its computational complexity, none of them deal with social interaction and spatial spill-over effects in disaggregate transportation behavior (Anselin, 1988; Fleming, 2004).

While there exists a spatially autoregressive mode choice model using aggregate data (Boldoc et al., 1995), a spatially autoregressive disaggregate mode choice model based on real-world, empirical data to examine network effects has never been done.

In the next section, I will lay out the theory of how to econometrically model network effects, followed by an overview of the data used and the issues surrounding the design of the weight matrix. Then, I develop the actual econometric models and evaluate the result before finishing with a discussion of the consequences for policy makers.

\section{THEORY OF MODELING NETWORK EFFECTS}

In Goetzke (2006) the theory of network effects is introduced as it applies to transportation mode choice decision making. Interpreting network effects as a signal that a certain transportation mode is safe and reliable ${ }^{7}$, the main claim of the paper is that the utility of taking this mode increases with its

\footnotetext{
${ }^{7}$ Just like a full restaurant is a sign for good food and satisfied customers.
} 
mode share. Therefore, the more people use the mode, the more attractive this transportation mode becomes for all other people. These social spill-over effects lead to positive demand-side network externalities, the exact opposite from what is known as congestion. ${ }^{8}$

However, Goetzke did not provide any empirical evidence that these network effects exist. In this paper, I develop a rigorous econometric framework to examine real-world data and quantify these network effects. The starting point for the analysis is the random utility conditional mode choice model, the traditionally used workhorse of transportation demand modeling and forecasting (McFadden, 1974; Ben-Akiva and Lerman, 1985; Train, 2003). Because of the structure of the spatial weight matrix used in the model, I will restrict the analysis in this paper to a binary mode choice model. However, the general approach can be extended to a multinomial mode choice model, as discussed in the conclusion.

Let's consider the utility $v_{n i}$ of $N$ individuals with $n=1, \ldots, N$ using mode $i$, which is a function of $A$ personal characteristics, $x_{a n}$, with $a=1, \ldots, A$, and the $B$ mode-specific characteristics, $t_{b i}$, with $b=1, \ldots, B$. Assuming that $v_{n i}$ is a linear combination of $x_{a n}, t_{b i}$, as well as a random error term $\varepsilon_{n i}$

\footnotetext{
${ }^{8}$ While it is in this context possible to also analyze negative congestion externality, it is not the focus of this study.
} 
(stemming from unobserved utility determinants), an empirical utility model could be expressed as follows:

$v_{n i}=\alpha_{1} x_{1 n}+\ldots+\alpha_{a} x_{a n}+\beta_{1} t_{1 i}+\ldots+\beta_{b} t_{b i}+\varepsilon_{n i}=x_{n} \alpha+t_{i} \beta+\varepsilon_{n i}$

where $x_{n}$ is a $1 \times A$ vector of personal characteristics, $t_{i}$ is a $1 \times B$ vector of mode characteristics, and $\alpha$ and $\beta$ are the corresponding column vectors of regression parameters.

However, data can only be collected on transportation mode choice decisions, not on mode-specific utility levels. Therefore, Equation (1) has to be transformed in such a way that $v_{\text {in }}$ becomes an unobserved, latent variable. Assuming that $\varepsilon_{n i}$ is logistically distributed, a binary logit mode choice model can be derived, with $P_{n}(i)$ being the probability of person $n$ to choose mode $i$ over mode $j$ :

$$
\begin{aligned}
P_{n i} & =P\left(v_{n i} \geq v_{n j}\right) \\
& =\left(\exp v_{n i}\right) /\left(\exp v_{n i}+\exp v_{n j}\right) \\
& =1 /\left[1+\exp -\left(v_{n i}-v_{n j}\right)\right]
\end{aligned}
$$


If the above random utility model is extended to account for network effects, it is necessary to add a spatially autogressive mode-choice term to Equation (1). Therefore, the model includes a spatial weight matrix, $W$, of the dimension $N \times N$, as well as an $N \times 1$ vector $m_{i}$ of revealed mode choice decisions by people. Both terms together, $\left(W m_{i}\right)$, result in the spatially weighted average mode share of mode $i$ for all the neighboring people of individual $n$. The scalar $\rho$ is the regression coefficient for the spatial lag term:

$v_{n i}=x_{n} \alpha+t_{i} \beta+\left(W m_{i}\right) \rho+\varepsilon_{n i}$

The spatially autoregressive structure becomes more visible when considering that $m_{i}$ is actually a function of the $N \times 1$ mode-specific utility vector $v_{i}$ for all individual $n$. If the mode choice model is now written down in its full matrix form, then the issue of spatial autoregression can be easily seen:

$$
\begin{aligned}
P\left(m_{i}=1\right) & =1 /\left[1+\exp -\left(v_{i}-v_{j}\right)\right] \\
& =F\left[x \alpha,\left(t_{i \neq 1}-t_{i=1}\right) \beta,\left(W m_{i}\right) \rho\right]
\end{aligned}
$$

Assuming that the error term does not exhibit any spatial autocorrelation, Equation (4) represents now what Anselin (2002) calls a 
conditional spatially autoregressive discrete choice model. The estimation of this model is straight forward, since a maximum likelihood approach can be used. The model design is also very similar to the one proposed by Páez and Scott (2006), except that the network effects are not based on social interaction, but rather on spatial dependency.

The $1 \times N$ vector of probabilities $P$ to use mode $i$ becomes a function of: the $N x A$ individual characteristics matrix $x$; the differences between the two $N \times B$ mode-specific characteristics matrices $t_{i \neq 1}$ and $t_{i=1}$, which are related to both available modes; the average mode share of all surrounding neighbors $\left(W m_{i}\right)$, representing network effects; and all the corresponding vectors of regression coefficients $\alpha, \beta$, as well as the regression coefficient scalar $\rho$. Because of the spatial weight matrix referring to just one mode, in its basic form this model can only be applied to binary choice models. I will discuss in the conclusion an extension towards multinomial choice models.

\section{Figure 3.1 about here}

Equation (4) essentially says that, with increasing neighboring mode share in mode $i=1$, the probability of a person to also choose this transportation mode increases as well. Therefore, a positive value of $\rho$, which 
is significantly different from zero, indicates the existence of network effects. Now, if the utility of using the mode is an indicator of the willingness to pay (WTP) and assumed to be correlated with the WTP for the transportation mode, the regression coefficient $\rho$ can be interpreted as the value of the slope for the upward-sloping WTP curve with respect to mode share, due to network effects (see Figure 3.1). However, in reality it is expected that different people have individual levels of $\rho$, which cannot be modeled as such, because the number of variables, including all the personal network preferences would surpass the available degrees of freedom. Therefore, the network effects estimate $\rho$ refers to the aggregate network effects.

\section{DATA SET AND WEIGHT MATRIX}

The data used for the spatially autoregressive logit mode choice model is based on the 1997/1998 comprehensive regional household travel diary survey conducted for the Best-Practice Travel Demand Forecasting Model by the New York Metropolitan Transportation Council (NYMTC). New York City seemed to be a very good choice for this analysis, because, while it is an American city, it also has a large transit ridership. These two facts make the 
results easier transferable to both different American and other cities in the world. $^{9}$

The data was collected for the whole metropolitan area of 28 counties, which include parts of upstate New York, New Jersey and Connecticut, but I only took data from the five New York City boroughs of the Bronx, Brooklyn, Manhattan, Queens and Staten Island. The main reasons for choosing these boroughs are:

- The New York City area has a relatively high density of surveyed households, allowing a meaningful weight matrix to be developed, which is necessary for estimating the autoregressive model. Outside of the NYC area, the distances between surveyed neighbors becomes rather large.

- Only in New York City is the number of transit riders high enough to get the variability in the data set desired for econometric analysis. Also, NYC has an integrated transit system. Therefore, individuals there face roughly the same transit infrastructure.

\footnotetext{
${ }^{9}$ The fact that the data set is almost 10 years old should not have any impact on the results, because of the general nature of this research.
} 
For the mode choice model, only trips from home to work were used. The literature in classical transportation modeling distinguishes between the three typical trip purposes: Home-based work trips (HBW), non home-based trips (NHB), and home-based other trips (HBO). Each trip purpose is modeled differently, since the mode choice determinants are expected to differ. Purely for practical reasons I decided to restrict my analysis only to HBW trips. I also included only transit and drive-alone trips (more than $90 \%$ of all HBW trips) between different travel analysis zones (TAZ) within New York City which were longer than 1.5 miles or $2.4 \mathrm{~km}$ (to exclude the possibility of walking). In the end I had 1,652 person trips from all five boroughs of New York City, of which 32.7 percent drove alone to work and the remaining 67.3 percent used transit.

Using U.S. census block group longitude and latitude information, Figure 3.2 displays the location of the surveyed households used to create the spatial weight matrix. As it can be seen in the map, the density of households is much greater in Manhattan compared to the remaining four boroughs. This can be contributed to the fact that the sample is not spatially random, since the sampling was not done with a spatially autoregressive model in mind.

Figure 3.2 about here 
Accounting for the heterogeneous household location density, a rowstandardized spatial weight matrix of the dimension $N x N$ was developed as follows: Each row contains the 40 closest neighbors of the corresponding observation $n$ which are equally weighted. Adding more than 40 neighbors would have not given significantly more information, since the average mode share will not change much anymore. As soon as a neighbor lives further away than 0.75 miles $(1.2 \mathrm{~km})$, however, he or she was not included, unless the number of neighbors would drop below four. The weight matrix needs a minimum number of neighbors in order to be meaningful. If a neighbor is further away than $0.75(1.2 \mathrm{~km})$, or a 20 minute walk, the neighborhood characteristics may change too much to reflect network effects. The sum of the weights in each row adds up to one, so that the spatial weight matrix effectively calculates the spatially moving transit mode split average.

This method of deriving a spatially moving transit mode split average represents a unifying decision rule, while it also allows accounting for the heterogeneity of the boroughs in terms of spatial household density. It turns out that the average number of neighbors is 23 , with the maximum number of 40 neighbors existing for about a quarter of the records. The average distance of 
neighbors is 0.4 miles $(0.64 \mathrm{~km})$ and very few of them, a little more than $1 / 2$ percent, are further away than $0.75(1.2 \mathrm{~km})$.

\section{MODEL AND RESULTS}

The objective for developing the following model is to find out whether or not network effects exist for transit use. The basic set-up is a McFaddentype mode choice model (McFadden, 1974) that includes the commonly used determinants of mode choice decision making: total travel time, access to a car, income, and gender. Age was not considered because of the large number of missing observations. Mainly to control for parking cost in Manhattan, a dummy was added as a proxi for all Manhattan destinations. Finally, the network effects for transit use was measured as the spatially autoregressive term of revealed mode choice decisions previously described, which essentially is equivalent to the spatially weighted average transit mode share of all neighbors. The model, however, accounts only for aggregate network effects. The expected individual network effects cannot be captured econometrically, due to the restricted number of degrees of freedom, as discussed above.

Three different models were evaluated. The first model, called the basic model, omits network effects and represents the traditional approach of mode choice modeling. In the following two models, network effects are included. 
The model with network effects incorporates a variable for the aggregate network effects. In the model with network effects controlled for car access, which was run to do sensitivity analysis, the network effects were split into an aggregate component and a component for people not having access to a car.

\section{Table 3.1 about here}

The results of all three models are exhibited in Table 3.1. Standard errors are shown in parentheses and statistical significance is indicated by stars. All estimated regression coefficients of the basic model have the expected signs. Except for the mode-specific constant and the high income group variable, they are also significantly different from zero at the one-percent level. While it is not a problem that the estimate of the regression coefficient for the high income group variable is not significantly different from zero, it is potentially a problem for the mode-specific constant term, since it is expected that people prefer the automobile over transit.

The regression coefficient of the total travel time variable is negative because the indirect utility derived from using any transportation mode decreases with more time spent on it. In this context it should be emphasized that the total travel time regression coefficient of the McFadden-type mode 
choice model is based on the difference of the mode-specific travel time, using the transportation mode in the constant term as the point of reference. Therefore, total travel time measures effectively how much it takes longer to ride transit in comparison to the car.

Obviously, if a person does not have access to a car, he or she is more likely to use transit. This explains the positive sign for the no-access-to-a-car dummy coefficient estimate. Since parking is very costly in Manhattan, people are more inclined to take transit there, which makes the regression coefficient positive. The automobile is a normal good, therefore, the high income group will avoid transit, resulting in a negative coefficient estimate. Finally, men are less willing to ride transit, leading to a negative regression coefficient.

The model with network effects improves upon the mode-specific constant (in comparison to the basic model), since the regression coefficient estimate, while keeping its negative sign, becomes now significantly different from zero. In addition, the new model reveals a positive aggregate network effects for transit use, while all the other coefficient values remain very robust. A negative network effects coefficient value would have been a sign of an aggregate personal aversion towards transit crowding which is typically known as congestion externality. 
It is interesting to interpret the constant term together with the network effects: With very low transit mode share, the relative indirect utility of using transit is smaller than for driving alone (see negative value of the constant). As soon as the transit mode share moves towards one, the difference of indirect utility between transit use and drive alone decreases until transit use utility eventually surpasses indirect utility of driving alone, since the network effects coefficient is larger than the coefficient for the constant. This is exactly what is expected from network effects. The willingness to pay for transit, which is related to the indirect utility, increases when more people take transit, and thus lowers the relative cost of using transit (vs. the car).

In the model with network total travel time is the major variable representing the supply side. For the transit mode, total travel time includes invehicle travel time, access and egress time, as well as wait time. For the drive alone mode, total travel time is based on the automobile travel speed. Therefore, total travel time is an aggregate variable of the physical infrastructure, accounting for transit service level and highway congestion.

However, what if the network effects variable captures a portion of the supply side? It can be seen in Table 3.1 that the coefficient estimates for total travel time are essentially the same in the basic model and the model with network effects. In the case of network effects incorporating the infrastructure, 
it is expected that the values would be not as close as they are. It could also be argued that the network effects variable accounts for some unmeasured supply level. For example, a person without having access to a car may live closer to a transit station and is more likely surrounded by people also without car access. Thus, better transit availability could easily explain network effects.

The model with network effects controlling for car access addresses the issue by splitting the network effects into two components: the aggregate network effects and the network effects for people without access to a car. The most important finding is that the inclusion of the second network effect component does not greatly change the value of the regression coefficient for aggregate network effects. Furthermore, the estimate of the network effects regression coefficient for people without access to a car is not significantly different from zero. If the network effects variable for people with no access to a car is analyzed in conjunction with the no-access-to-a-car dummy, it can be seen that the network effects coefficient absorbs part of the dummy coefficient. The dummy coefficient estimate becomes less significant and decreases at about the same value of the additional network effects regression coefficient for people without access to a car, multiplied by the average transit mode share. All other coefficient estimates remain stable in value and significance. The attempt of the last model to control for potentially unobserved supply side 
effects that stem from the physical infrastructure did not yield any improvements. The original model with network effects can be viewed as robust.

It still may be possible that the network effects variable captures some effects of the transit amenities such as transit safety, crime rates, station cleanliness or infrastructure quality. Furthermore, using transit in high numbers may even generate these amenities, when, for example, transit riders create a less crime-ridden environment, or demand a cleaner transit station. This is exactly what Goetzke (2006) discussed as the possible source of network effects, when he views them as a signal for safe and reliable transit. Therefore, unlike in the typical mode choice model, all these formerly omitted transit characteristics can now conveniently be seen as summarized in the network effects variable. In this context, the network effects variable gives way to a nice interpretation: It essentially becomes an indication of people's perception how well transit works.

Now it can be concluded that in the presence of network effects, the individual mode choice decision making does not only depend on personal traits and mode-specific characteristics, but also on the mode share of the person's neighborhood. This means, everything else being equal, and counter to conventional wisdom, that poor people are less likely to use transit in areas 
with low transit share than in areas with high transit share. On the other hand, more wealthy people take transit in areas with high transit share compared to areas with low transit share.

\section{POLICY CONSEQUENCES}

The fact that transit use exhibits network effects may lead to farreaching policy consequences. It is typical for both transportation planners as well as policy decision makers to depend on travel demand forecasting models for evaluating new transit projects. The centerpiece of every travel demand forecasting model is the mode choice model, which traditionally does not include a network effects variable to account for spatial autoregression. This non-inclusion of network effects in the model formulation causes an omitted variable bias which becomes visible in the mode-specific constant term, as shown in Goetzke (2003). The result is, therefore, a systematic forecasting error where transit ridership in suburbs with low transit mode share are overestimated and transit ridership in the central city with high transit mode share are underestimated.

In the traditional mode choice model approach, with everything else being equal, the mode-specific constant term for transit use captures the difference in indirect utility between transit use and the alternative mode (in 
this case: drive-alone trips). If this mode-specific constant term is not allowed to change in space, a person in the suburbs with low transit ridership will be forced to have the same average differential indirect utility for transit use over driving alone as the person living in the central city with high transit ridership. However, in reality, network effects are responsible for the fact that a person living in a transit-unfriendly suburb derives less differential utility from choosing transit over driving with the car, than the person living in the transitfriendly city.

It should now be easy to see that as long as the differential utility for using transit in the suburbs is lower than average, and in the central city, higher than average, transit ridership will be overestimated in the suburbs and underestimated in the central city. Figure 3.3 demonstrates graphically how not including the network effects variable into the model leads to an omitted variable bias in the regression.

\section{Figure 3.3 about here}

This new insight might at least partially explain why new rail starts in the past decade have had problems with inflated ridership forecasts compared to the observed ridership after opening (Pickrell, 1989; Kain, 1992). Since the 
federally funded rail projects studied by Pickrell serve mostly the commuter market from the suburbs to the $\mathrm{CBD}$, the systematic bias in the mode choice model caused by unaccounted for spatial autoregression stemming from network effect could be responsible for overestimating ridership. Not accounting for network effects may have also dwarfed the forecasts of Tren Urbano in Puerto Rico, which is in 2005 less than a third of the originally expected ridership (Green, 2005).

\section{CONCLUSION}

I have shown that network effects play a role in mode choice decision making. However, the theory only allows for spatially autoregressive binary mode choice models because of the spatial weight matrix character $(\mathrm{Wm} *)$, with $m^{*}$ taking on the value of either zero or one, but never a larger number. Given what was discussed in this paper about spatially autoregressive binary mode choice models, it is not difficult to extend the general concept to multinomial or even nested mode choice models. The spatial lag term representing the network effects is just the average mode share of all the spatially weighted neighbors. By replacing the social interaction with spatial dependency, this concept can easily be included in the model structure proposed by Páez and Scott (2005). 
An extension of the mode choice model would be to not only include network effects on the origin side of the trip, but also on the destination side of the trip. Intuitively, this makes a lot of sense since people make their mode choice decision based on both origin characteristics and on destination characteristics. LeSage and Pace (2005) find an elegant solution for designing the appropriate spatial weight matrix.

While the estimation of a spatially autoregressive mode choice model is feasible as long as the data is collected on the basis of spatial sampling, the real challenge poses the inclusion of network effects in mode choice forecasting for new infrastructure investment projects. In traditional mode choice models, the mode share is exclusively determined by the social characteristics as well as trip costs - both being assumed to be exogenous. Network effects, however, lead to the fact that a portion of the trip cost (WTP for using the mode with others) itself becomes endogenous. At the same time, one does not know what the individual preference for using the transportation mode together with other people would be.

This problem is somewhat similar to the issue of congestion in the mode choice model, where a portion of the trip cost (congestion cost) also becomes endogenous. As part of the travel time variable, it is assumed that the congestion cost effects everyone the same way as an average congestion cost 
does. When faced with this problem, the typical resolution for deriving model forecasts is a simulation approach, where the model results are iteratively looped until convergence is reached.

In the context of network effects, the simulation approach assumes that socio-economic characteristics are exogenous and will not change with the implementation of new transit facilities, which essentially means that improved public transit triggers nobody to move. Such an assumption is unrealistic, since the social composition of a neighborhood is, of course, endogenous with respect to transportation infrastructure. This problem even exists for traditional mode choice models, but may be alleviated by including a land-use model. However, network effects do magnify the problem. People move into the neighborhood because of the new transportation infrastructure and will use this mode now available. In addition, the new residents will also trigger already older residents to switch to the newly available transportation mode. Therefore, a mode choice model that includes network effects has to always integrate a land-use model when deriving forecasts.

In the context of the four-step transportation model, I am convinced that not just the mode choice step is spatially autoregressive, but also the trip generation model and the trip assignment model. LeSage and Polasek (2005) discuss the possibility of spatial autoregression in the trip distribution model. 
Naturally, people in the central city make more, but shorter trips than their suburban counterparts. They also tend select their destinations, i.e. shopping, based on the destinations of others. Especially in congested cities, people might even choose their routes on what is conceived as being the fastest way, but may actually become slower because of these route choice network effects causing congestion. After all, the actual decision making in difficult traffic conditions will always lack full information with the result that assignment flows may be in a continuous disequilibrium, or in equilibrium, but with network effects.

\section{REFERENCES}

Anselin, L. (1988) Spatial Econometrics: Methods and Models. Dodrecht, NL and Boston, MA: Kluwer Academic Publishers.

Anselin, L. (2002) Spatial Regression Analysis Short Course. Ann Arbor, MI: University of Michigan (unpublished manuscript).

Ben-Akiva M. and S. Lerman (1985) Discrete Choice Analysis: Theory and Application to Travel Demand. Cambridge, MA and London, UK: MIT Press.

Boldoc D., R. Laferriere and G. Santarossa (1995) Spatial Autoregressive Error Components in Travel Flow Models: An Application to Aggregate 
Mode Choice, in: L. Anselin and R. J. G. M. Florax (eds.) New

Directions in Spatial Econometrics. Berlin, Germany: Springer Verlag, pp. 96-108.

Brock, W. and S. Durlauf (2001) Discrete Choice with Social Interactions, Review of Economic Studies, 68, pp. 235-260.

Brock, W. and S. Durlauf (2002) A Multinomial-Choice Model of Neighborhood Effects, American Economic Review, 92, pp. 298-303.

Fleming, M. (2004) Techniques for Estimating Spatially Dependent Discrete Choice Models, in: L. Anselin , R. Florax and S. Rey (eds.) Advances in Spatial Econometrics. Berlin, Germany: Springer Verlag, pp. 145-168.

Goetzke, F. (2003) Are Travel Demand Forecasting Models Biased because of Uncorrected Spatial Autocorrelation? Regional Research Institute Working Paper 10.

Goetzke, F. (2006) Mode Choice and Social Networks: The Economics of Walking, Bicycling and Public Transit Use. Morgantown, WV: West Virginia University (dissertation essay).

Green, A. (2005) A Hesitant Puerto Rico Tries Commuting by Train, The New York Times, November 19th. 
Kain, J. F. (1992) The Use of Straw Men in the Economic Evaluation of Rail Transport Projects, American Economic Review, 82, pp. 487-493.

LeSage, J. P. and R. K. Pace (2005) Spatial Econometric Modeling of OriginDestination Flows. Las Vegas, NV: 52st Annual North American Meetings of the Regional Science Association International.

LeSage, J. P. and W. Polasek (2005) Incorporating Transportation Network Structure in Spatial Econometric Models of Commodity Flows. Toledo, OH: University of Toledo (unpublished paper).

McFadden, D. (1974) Conditional Logit Analysis of Qualitative Choice Behavior, in: P. Zarembka (ed.) Frontiers in Econometrics. New York, NY: Academic Press, pp. 105-162.

Páez A. and D. M. Scott (2005) A Discrete Choice Approach to Modeling Social Influence on Individual Decision Making. Seattle, WA: 51st Annual North American Meetings of the Regional Science Association International (revised version of the presentation).

Páez A. and D. M. Scott (2006) Social Influence on Travel Behavior: A Simulation Example of the Decision to Telecommute, Environment and Planning A, forthcoming. 
Pickrell, D. H. (1989) Urban Rail Transit Projects: Forecasts vs. Actual Ridership and Costs. Cambridge, MA: U.S. Department of Transportation, Transportation System Center.

Train, K. (2003) Discrete Choice Methods with Simulation. Cambridge, UK and New York, NY: Cambridge University Press. 
Figure 3.1: Positive network effects for an individual person in their mode choice decision.

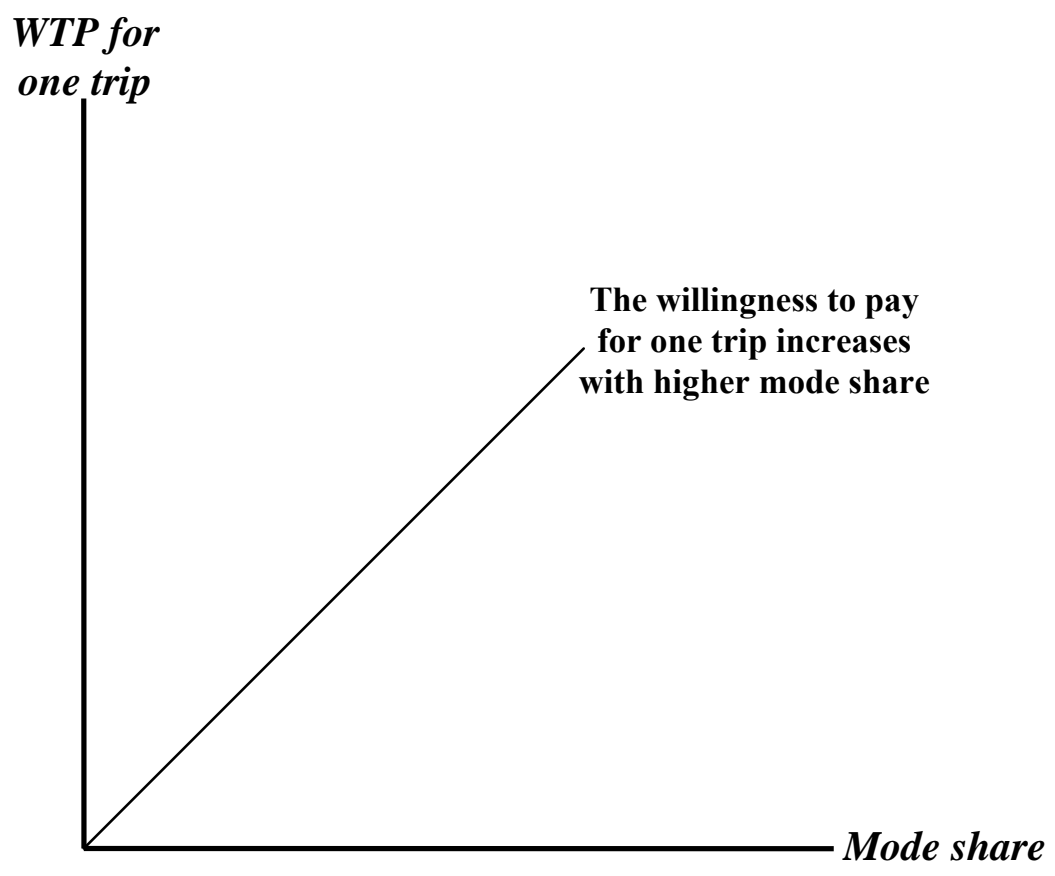


Figure 3.2: Geo-coded locations of all surveyed households in New York City.

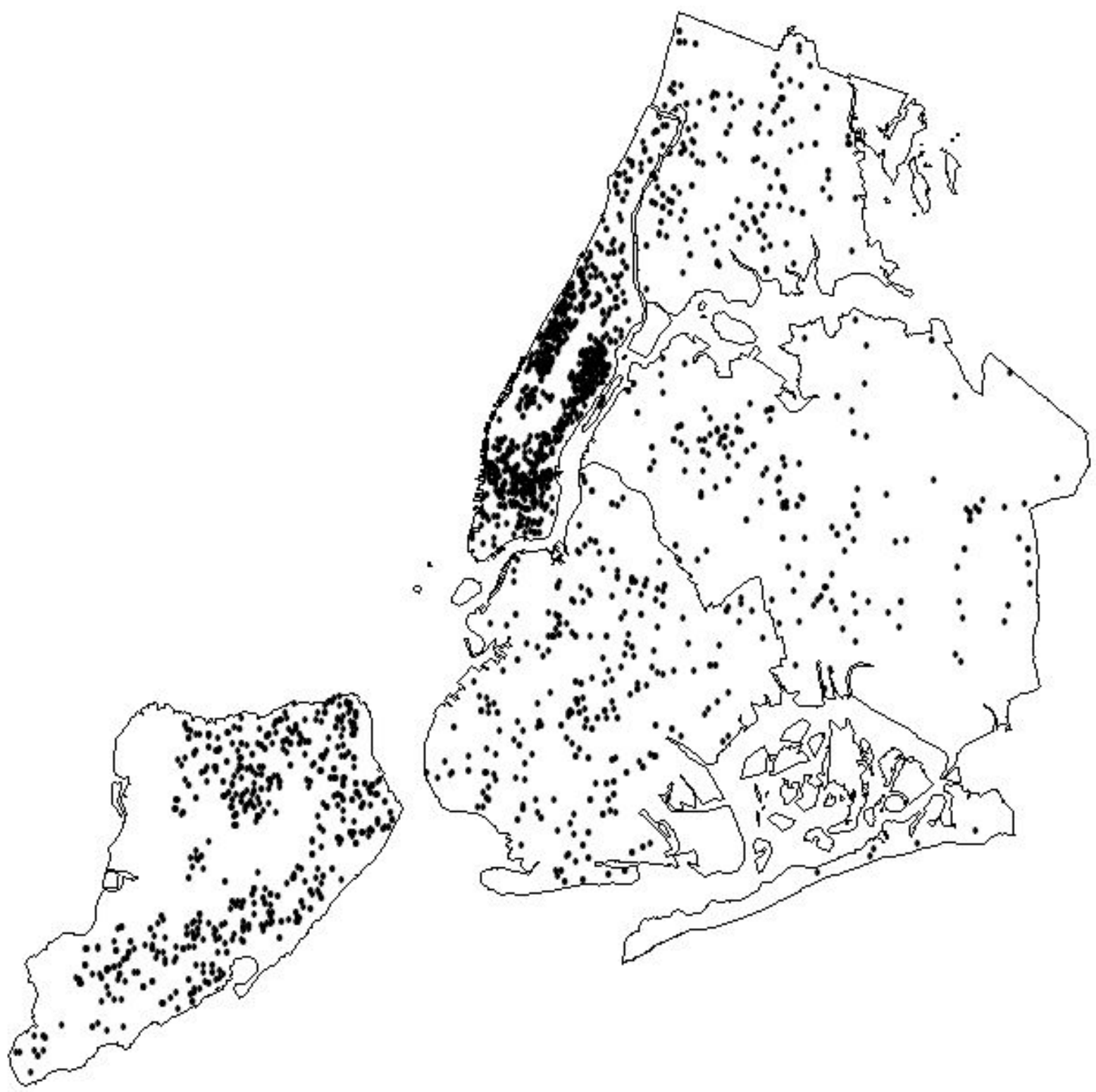


Table 3.1: Estimation results for the New York City mode choice model.

\begin{tabular}{|c|c|c|c|}
\hline & Basic Model & $\begin{array}{c}\text { Model with } \\
\text { Network Effects }\end{array}$ & $\begin{array}{l}\text { Model with Network Effects } \\
\text { Controlling for Car Access }\end{array}$ \\
\hline $\begin{array}{r}\text { Transit Mode Constant } \\
(\text { drive alone }=0, \text { transit }=1)\end{array}$ & $\begin{array}{l}\mathbf{- 0 . 0 7 9} \\
(0.194)\end{array}$ & $\begin{array}{c}-\mathbf{1 . 0 4 7 * *} \\
(0.248)\end{array}$ & $\begin{array}{c}\mathbf{- 1 . 0 0 1 * *} \\
(0.250)\end{array}$ \\
\hline Total Travel Time $(0,1)$ & $\begin{array}{c}\mathbf{- 0 . 0 3 6 * *} \\
(0.006)\end{array}$ & $\begin{array}{c}\mathbf{- 0 . 0 3 5 * *} \\
(0.006)\end{array}$ & $\begin{array}{c}-\mathbf{0 . 0 3 6 * *} \\
(0.006)\end{array}$ \\
\hline $\begin{array}{r}\text { Average Network } \\
\text { Effect for Transit Use (1) }\end{array}$ & & $\begin{array}{l}1.887 * * \\
(0.293)\end{array}$ & $\begin{array}{l}\mathbf{1 . 8 1 3} * * \\
(0.296)\end{array}$ \\
\hline $\begin{array}{l}\text { Additional Transit Network } \\
\text { Effect for No Car Access (1) }\end{array}$ & & & $\begin{array}{c}2.174 \\
(1.591)\end{array}$ \\
\hline No Car Access Dummy (1) & $\begin{array}{c}4.258 * * \\
(0.398)\end{array}$ & $\begin{array}{l}3.893 * * \\
(0.404)\end{array}$ & $\begin{array}{l}2.463 * \\
(1.041)\end{array}$ \\
\hline Destination Manhattan (1) & $\begin{array}{l}1.991 * * \\
(0.201)\end{array}$ & $\begin{array}{l}1.831 * * \\
(0.207)\end{array}$ & $\begin{array}{l}1.819 * * \\
(0.207)\end{array}$ \\
\hline High Income Dummy (1) & $\begin{array}{l}\mathbf{- 0 . 2 4 2} \\
(0.157)\end{array}$ & $\begin{array}{l}\mathbf{- 0 . 3 1 1} \\
(0.161)\end{array}$ & $\begin{array}{l}\mathbf{- 0 . 3 0 9} \\
(0.161)\end{array}$ \\
\hline Male Dummy (1) & $\begin{array}{c}\mathbf{- 0 . 7 0 2 * *} \\
(0.159)\end{array}$ & $\begin{array}{c}\mathbf{- 0 . 7 3 5 * *} \\
(0.163)\end{array}$ & $\begin{array}{c}\mathbf{- 0 . 7 3 0 * *} \\
(0.163)\end{array}$ \\
\hline Sample Size & 1,652 & 1,652 & 1,652 \\
\hline Log-likelihood & -534.03 & -512.75 & -511.80 \\
\hline Pseudo- $\mathbf{R}^{2}$ & 0.528 & 0.546 & 0.546 \\
\hline
\end{tabular}

*significant at the $5 \%$-level

**significant at the $1 \%$-level 
Figure 3.3: In the presence of network effects transit utility increases with higher transit mode share. However, if network effects are not included into the model, transit utility is forced to be constant, which leads to an omitted variable bias. Therefore, transit ridership will be systematically underestimated in the suburbs $\left(\mathrm{v}_{1}<\mathrm{v}_{2}\right)$, and overestimated in the central city $\left(v_{1}>v_{2}\right)$.

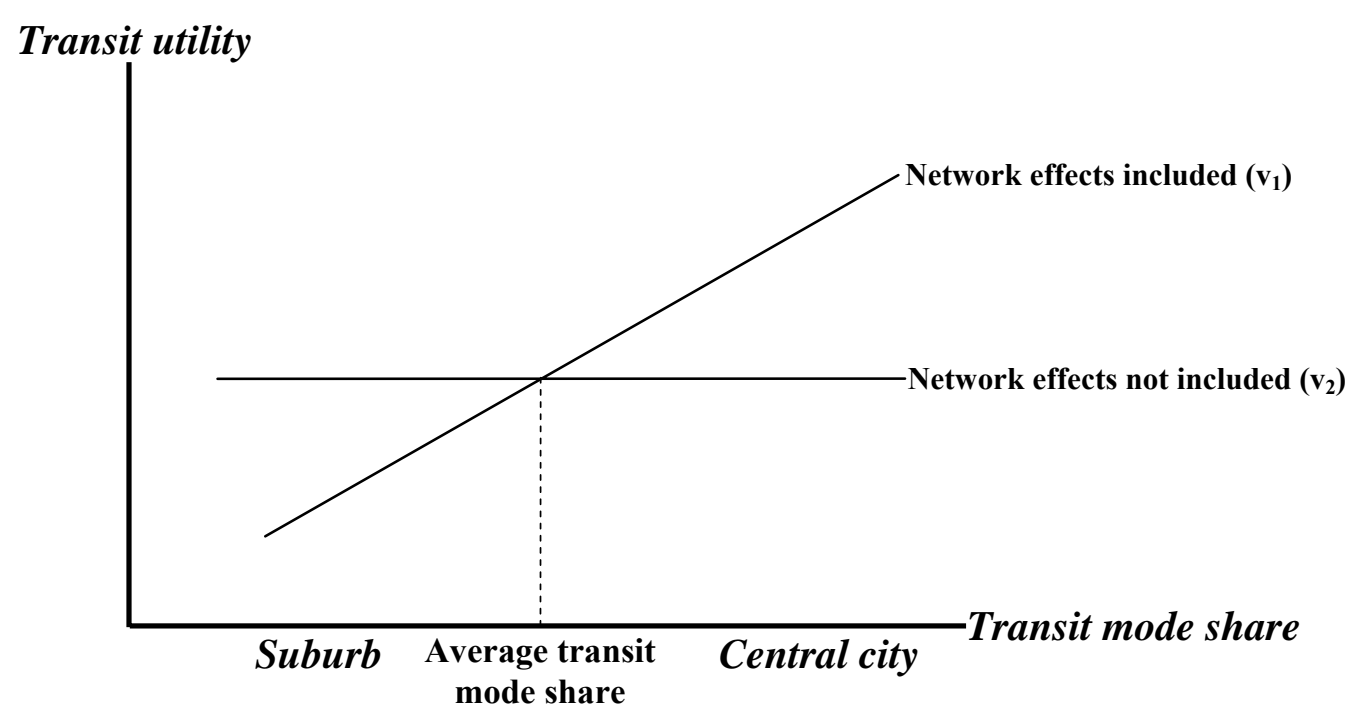




\section{Chapter 4}

\section{Bicycle Use in Germany: Explaining Differences}

between Municipalities with Social Network Effects 


\section{INTRODUCTION}

The bicycle modal split in German cities differs significantly. It ranges from a low of less than five percent in the state capital, Wiesbaden, to a high of more than one-third in the university town, Münster. Conventional wisdom would refer to Wiesbaden's hilliness to explain the small number of bicyclists, and to Münster's young average age in Münster to explain the opposite pattern there. A city's physical environment (i.e. its topography, infrastructure, socioeconomic composition, including age and the trip characteristics) plays a dominate role in determining how many people use their bike. The results of Siu et. al. (2000) and Rieveld (2004) support the importance of these municipal traits. In this paper, I apply a binary discrete choice model, which includes not only these traditional variables, but also a measure of the city's "biking culture". I find that the probability of a person riding a bicycle increases with the city’s level of “biking culture”.

“Biking culture” is best described as a social interaction or spill-over phenomenon, where a larger number of bicyclists make it more likely that some other person will also ride a bike. In the literature, this subject matter is discussed as neighborhood or social network effects (Shy, 2001; Goetzke, 2006a). In the last few years innovative econometric approaches were developed to empirically test for these social network effects (Brock and 
Durlauf, 2001; 2002; Paez and Scott 2005; 2006; Dugundji and Walker, 2005; Goetzke 2006b). This study is not only the first bicycle choice model for Germany, ${ }^{10}$ but also the first to use municipal-level social network effects reflecting "biking culture" to explain bicycle modal share differences across cities. ${ }^{11}$ If social network effects indeed play a significant role in whether or not people choose a bike as their transportation mode, then approaches to bicycle policy may shift to building and supporting a "biking culture" rather than exclusively to improving infrastructure. In general, bicycle infrastructure results from biking culture, while good bicycle infrastructure does not necessarily improve biking culture.

The next section provides an overview of the data used and develops an explanatory framework. Then I introduce a methodology to capture biking culture through social network effects and develop an econometric model. Finally, I finish with a policy discussion and conclusion.

\footnotetext{
${ }^{10}$ All German bicycling studies are restricted to either qualitative or univariate statistical analysis.

${ }^{11}$ As of now, no mode-choice model has dealt with city-wide network effects.
} 
PREVIOUS LITERATURE, EXPLANATORY FRAMEWORK, AND DATA

Using data from the 2002 nationwide survey of travel behavior, “Mobility in Germany - MiG 2002” (BMVBW, 2003), I build a binary logistic regression model to analyze municipal differences in bicycle mode choice decision making.

Few regression models have been developed to explain the difference in bicycle use between cities within a country. For Germany, just two studies compare bicycle behavior across cities (Pez, 1998, Flade et. al., 2002), but neither employs a regression model. Rietveld and Daniel (2004) used an aggregate regression model with data from 103 Dutch municipalities. They found that besides socio-economic and demographic variables, weather and bicycle infrastructure have statistically significant impacts on bicycle mode share. In the only discrete choice model looking at municipal differences in the bike modal split, Siu et. al. (2000) determined that a topography dummy variable is positive and significantly different from zero. Rave (2005) provides an excellent overview of the empirical bicycle studies.

Explanatory variables used in these regression models, as well as in other research papers, such as single-city bike choice models (Ortuzar et. al., 2000), can be grouped into three categories: 
1. Individual characteristics: Sex, age and income are considered the most important personal variables to determine bicycle use. Males are typically more likely to use a bike, while bicycle riding decreases with age. Poor people tend to ride their bike more often.

2. Trip characteristics: Trip length and weather are the typically included determinants of bicycle use. Longer trips, and bad weather are not conducive to biking.

3. Municipal characteristics: Empirical evidence points to biking infrastructure and topography as having a significant impact on bicycling. People are more likely to bike if the biking infrastructure is good and if the topography is flat. In some aggregate regression analyses, such as in Rietveld and Daniel (2004), other municipal characteristics, e.g. city size and density are used as a proxy for average trip length. In this study, which uses a discrete choice model, the inclusion of trip length renders these variables as unnecessary. 
The MiG 2002 provides most of the data described above. The basic sample has 25,000 household records, 61,000 person records and 180,000 trip records. The additional regionally extended samples (Aufstockungstichprobe) bring the total up to approximately 50,000 households, 100,000 persons, and 400,000 trips.

Using the extended samples for the state of Nordrhein-Westfalen (Northrhine-Westphalia), as well as for the metropolitan area of Frankfurt, the region of Hannover, as well as the transportation districts of Nordhessen (North Hesse) and Bremen/Niedersachsen (Lower Saxony), I could identify a total of 32 cities larger than 50,000 inhabitants. The dataset with these 32 cities had more than 30,000 trips, which was reduced to around 10,000 trips after trips of persons less than 15 years old, weekend trips, trips longer than $100 \mathrm{~km}$, business trips, and return trips were removed. However, I could derive bicycling infrastructure proxies for only 24 of the 32 municipalities, which brought the final sample size to 8,725 , with each city subset having at least 100 trip records. Table 4.1 exhibits the summary statistics, as well as the source for all variables included in the model.

Table 4.1 about here 
Included variables describing individual characteristics are male, age, and low income. Since I have only information on income groups, I have aggregated the three lower income groups (monthly net household income less than 1,500 Euro) into a "poor" dummy variable. This dummy variable represents a bit more than one-eighth of the population, which coincides with the German poverty data.

Our model uses three variables, trip length, bad weather, and trip purpose for trip characteristics. A bad weather dummy was set to one if the conditions reported in MiG were either rain or snow. The trip purpose was split up into five categories: work trips, educational trips, shopping trips, leisure trips and all other trips (includes doctor visits, running an errand, etc).

The three variables included as municipal characteristics are topography, biking infrastructure and social network effects. None of these variables were part of the MiG survey. A flat topography dummy was set to one as long the city center and most of the area within the city did not appear as hilly by looking at topographical maps. I verified the results by telephone interviews with city officials. The flat topography values for each city are reported together with the other important municipal characteristics in Table 4.2 . 


\section{Table 4.2 about here}

This dataset only has information about travel time and distance for the chosen mode, but not for all the alternative modes, as is typically for McFadden-type travel demand models (McFadden, 1974). Thus, a different approach had to be found to control for each municipality's unique infrastructure. To avoid multicollinearity between variables, the additional challenge was to find an infrastructure variable that does not correlate with the social network effects variable (we discuss this issue in the next section). Two different approaches were used to derive a social network effects variable:

1. We conducted a telephone survey for 20 cities to find the bike lanes length in each municipality (in $\mathrm{km}$ ). This value is divided by the population to calculate the bike lane length per 1,000 inhabitants. It would have been preferable to compute bike lanes per area, but in Germany the municipal boundaries are politically determined and do not reflect the urbanized area. A city's reported area often includes small, annexed villages where most of the land use is for agriculture or forestry. 
2. In 2005, the German Federation of Bicyclist (ADFC) undertook a survey where people evaluated the bicycle infrastructure in their city (ADFC, 2005). Using the mean of both, accessibility for infrastructure quantity and average speed for infrastructure quality, I aggregate a bike infrastructure score which is ranging between a low of 0 for the worst infrastructure and a high of 5 for the best infrastructure. However, the data was available for only 17 cities.

While the "Bike Lane per Capita" variable seems to be more objective, bicyclists typically make decisions based on a personal, highly subjective assessment of the bicycle infrastructure. This may be better captured in the "Infrastructure Score" variable. The infrastructure proxies are highly correlated with each other, having for the 13 common cities in the two datasets a correlation coefficient of 0.675 , which is significant at the 2-percent level. This result is some indication that both infrastructure variables may capture essentially the same effects.

\section{METHODOLOGY AND ECONOMETRIC MODEL}

Goetzke (2006a) introduces the theory of social network effects as it applies to transportation mode choice decision making. Interpreting social 
network effects as a signal that biking is safe and reliable, ${ }^{12}$ the paper's main claim is that the utility of taking a bicycle increases with its mode share. Therefore, the more people using a bike, the more attractive a bike becomes to all other people. These social spill-over effects lead to positive demand-side network externalities, which I also refer to as biking culture in this paper.

In the last few years, several articles developed different approaches to econometrically model these social network effects. Based on the theoretical work by Brock and Durlauf (2001; 2002), Dugundji and Walker (2005) and Goetzke (2006b) used a social network effects approach to model travel demand. While Dugundji and Walker took zonal modal spit averages as a proxy for social network effects, Goetzke extended this approach to a spatially autoregressive mode choice model with moving mode share averages. Because of the different nature of the dataset, which includes more than one municipality, neither of these two methods is applicable for this paper.

In the spirit of the previous papers, I could have taken the average modal split for bicycling in each municipality as the proxy for social network effects. Because of the endogeneity of the biking culture proxy, however, this approach may exhibit a correlation between the social network effects variable and the error term, leading to a biased regression coefficient estimate.

\footnotetext{
${ }^{12}$ Just like a full restaurant is a sign for good food and satisfied customers.
} 
Therefore, the model requires an instrument that is correlated with the social network effects variable but not with the error term.

To create such an instrument, I have taken the trip purpose category (work and educational trips, shopping trips, leisure trips, and all other trips) and removed one trip purpose at a time from the dataset to get four different subsets. Then, the bicycle mode share derived from the excluded trip purpose records could be used as the instrument for the social network effects.

A total of four new datasets were generated: one excluding work and educational trips (bike mode share of work and educational trips is the social network effects variable); one excluding shopping trips (bike mode share of shopping trips is the social network effects variable); one excluding leisure trips (bike mode share of leisure trips is the social network effects variable); and one excluding all the other trips (bike mode share of other trips is the social network effects variable). The first column of Table 4.3 shows the correlation coefficients between the instrumental variables for biking culture and the total municipal bicycle mode share, an indicator of social network effects used in previous studies.

Table 4.3 about here 
The social network effects instrument captures municipal biking culture only as long as they are neither correlated with an omitted variable, nor with an included variable, such as biking infrastructure. Ignoring the infrastructure variable should upwardly bias the network coefficient because of the positive correlation between social network effects and infrastructure (omitted variable bias). On the other hand, if the infrastructure proxy is highly correlated with the social network effects variable, then coefficients of both variables may be adversely affected by multicollinearity.

Column 2 and 3 of Table 4.3 report the correlation coefficients between the social network effects proxies and the two infrastructure variables introduced in the previous section. The bicycle modal split for work and educational trips is correlated with the infrastructure score at the one percent significance level, the bicycle modal split for shopping trips is correlated with the infrastructure score at the five percent level, and four other correlations are significant at the ten percent level.

When I combine all three groups of determinants for bicycling choice, municipal, trip, and individual characteristics, a structural form of the binary logistic regression model can be derived as follows: 


$$
\begin{aligned}
\text { Bike }= & \alpha+\beta^{*} \text { individual characteristics }+\gamma^{*} \text { trip characteristics } \\
& +\delta^{*} \text { municipal characteristics }+\varepsilon
\end{aligned}
$$

Equation (1) ties together all the previous information. Male, age and the poverty dummy were used as individual characteristics. Included trip characteristics were trip length, a bad weather dummy, as well as the trip purpose (work, educational, shopping, leisure and other trips). Finally, the social network effects instruments, either of the two infrastructure proxies, and a flat topography dummy were taken as municipal characteristics.

In order for the regression estimation to be unbiased, the error terms should not be correlated with any of the independent variables which is a reasonable assumption. The social network effects instrument will actually capture all the remaining city-level autocorrelation in the error term, while, at the same time, not being correlated with the error term, because it is an exogenous instrument (bike mode share of the excluded trip purpose).

Another assumption of the model set-up is that there is no self-selection of the residential location. Since I analyze the city as a whole, and not specific neighborhoods, this assumption is expected to be met since few people will be choosing a city to live in just because of its biking culture. In this context, it may be also important to mention, that the dataset includes only trips where the bicycle is used predominately as a mode of transportation, not as a 
recreational tool. All the trips which recorded leisure as the trip purpose refer to the leisure activity undertaken at one of the trip ends.

At this point, however, the reader needs to be cautioned about one issue. Each municipality has a different level of biking culture represented in the social network effects variable. The regression coefficient for the social network effects estimates just the average impact of this biking culture on bicycle mode choice for all the cities combined. Unfortunately, a city-specific coefficient estimate is statistically impossible to obtain because the social network effects instruments would essentially become a municipal dummy. It would account for the average effect of all omitted city-level variables that would otherwise enter into the error term.

\section{MODEL AND RESULTS}

We evaluated eight models, two subgroups with four different data sets, respectively. The first subgroup includes bike lanes per capita for 20 municipalities as the infrastructure proxy, while the second subgroup uses the bike infrastructure score existing for 17 cities. Each of the four different data sets excludes one trip purpose (work and educational, shopping, leisure or other trips) so that the bike mode share for the excluded trip purpose could be used as the social network effects instrument. Tables 4.4(a) and 4.4(b) present 
the regression results. Most regression coefficients are significantly different from zero at the five-percent level or better and, with one exception discussed below, the signs are as expected.

\section{Tables 4.4(a) and 4.4(b) about here}

In all eight models, the social network effects regression coefficients have the expected positive sign, and are significantly different from zero at the one-percent level. This is a strong indication that municipal biking culture plays an important role in bicycle mode choice decision making. Therefore, a strong biking culture raises the probability that a person will ride the bike, ceteris paribus.

As seen in Table 4.4 (a), the regression coefficients for bike lanes per capita also have the expected positive sign, and are significantly different from zero at the ten-percent level or better. But if the infrastructure variable is used instead, the sign of the regression coefficient in Model (5), which excludes work and school trips, becomes unexpectedly negative at the one-percent

significance level. All other regression coefficients are still positive and significantly different at the one-percent level. 
The "wrong" sign for the bike lane coefficient in Model (5) may be explained by multicollinearity between the infrastructure measure and social network effects proxy, which are both correlated with each other at the onepercent level (see Table 3). This multicollinearity will likely result in the infrastructure score picking up some remaining effects from an omitted variable.

The effects of all the other included variables are as follows. Flat topography is more conducive to biking, as seen in the corresponding positive sign of the regression coefficient, which is in all cases significantly different from zero at the five-percent level or better. The longer the trip, the fewer people ride the bike. This relation ship is expressed in the trip length regression coefficient, which is always negative at the one-percent significance level. In bad weather, people are less likely to use their bicycle, causing the corresponding regression coefficient to have a negative sign which is significantly different from zero at the one-percent level at all times.

If compared to the base trip purpose (which is leisure in three models, and other trips in the remaining one), people prefer to ride the bike to work and especially to educational destinations (positive signs for both the work and educational trip coefficients are always significantly different from zero at the one-percent level). For all other trip purposes the regression coefficient is not 
significantly different from zero at the ten-percent significance level. Only in Model (8) is the shopping trip regression coefficient negative, and significantly different from zero at the five-percent level, making it a less preferred bike trip compared to the base trip purpose (leisure trip).

Males are more likely to use the bike, signified by a positive regression coefficient, which is always significantly different from zero at the one-percent level. On the other hand, age never has a statistically significant effect on bicycle riding. Finally, in two of the models using bike lanes per capita as the infrastructure measure, the poverty dummy has a significant positive effect at the five-percent level, providing some evidence that biking is an inferior good. Multicollinearity may explain the low level of significance of the age and poverty coefficient estimate. Both variables are correlated with the trip purpose categories. For example, work trips are undertaken by people who are typically not poor, and people taking educational trips tend to be young.

The magnitude of the regression coefficients estimated do not change between the eight models, and, in most cases, the actual values are very close together. This result supports the fact that the model specification is robust, especially given the somewhat different set of municipalities the two subgroups cover. 
In summary, the regression results show that social network effects, such as a varying biking culture in different cities, play an important role in determining a person's probability to ride the bicycle. The outcome, however, hinges on the assumption that the models control sufficiently for all other effects, especially the ones positively correlated with social network effects, such as infrastructure. Given the robustness of the model's regression coefficient estimates for social network effects, I believe that this assumption is a reasonable one.

\section{POLICY DISCUSSION}

Transportation planners typically believe that the most important policy for increasing bicycle mode share is to improve biking infrastructure (Nelson and Allen, 1997). The basic argument is that better facilities will lower the cost, and hence will increase the consumption of biking. In general, the model supports this policy approach, as the positive marginal effects of the infrastructure proxies reported in Tables 4.5(a) and 4.5(b) show.

\section{Tables 4.5(a) and 4.5(b) about here}


Because of the easier interpretation, only bike-lanes-per-capita is used for the discussion of the infrastructure variable. Depending on which trip purpose is excluded, its marginal effects range between 0.05 and 0.10 , with an average of 0.075 . This means that for a city of 100,000 inhabitants, building 1 $\mathrm{km}$ of bike lanes (or $0.01 \mathrm{~km}$ per 1,000 capita) will add 0.00075 to an average person's probability to ride the bike, or 0.075 percentage points. In other words, if a city invests in about $13.3 \mathrm{~km}$ of new bike lanes, the bike choice probability would go up by approximately one percentage point, say from 10 percent to 11 percent.

The model also included a second policy variable, social network effects capturing aspects of biking culture. Its marginal effects are shown in Tables 4.5(a) and 4.5(b). The values for the marginal effects for social network effects lie between one-third and two-third, again depending on which trip purpose is excluded.

The excluded trip purpose can be used to identify the relative dependence of each trip purpose on social network effects and which one less. Again, only the dataset with the bike-lane-per-capita variable is used for ease of interpretation. The two models with the lowest marginal effects for the network instruments are the ones excluding other trips (0.34) and shopping trips (0.46). This means the trip purposes that depend most heavily on social 
network effects are other trips and shopping trips. The trip purposes most independent of social network effects are work and education trips, as well as leisure trips, since the marginal effect of the datasets excluding these two trip purposes were 0.53 and 0.65 , respectively.

It makes sense that social network effects are not so important for leisure trip. People take their bicycle for recreation, because they enjoy it and not because they see other people riding their bike. While social network effects become more important for work and educational trips, they still do not have such an impact if compared to shopping and other trips. The reason may lie in the fact that for work and educational trips people evaluate the bike mode in comparison to their other alternatives whose cost can be significantly higher as long as congestion and parking for the automobile are included (infinite for people who do not have access to a car, such as young people with an educational destination). And public transit may make the trip much longer if access, wait, transfer and egress times are accounted for, in addition to the cost of being sometimes unreliable.

It is not at all surprising that shopping and other trips are the most sensitive to social network effects. People may only get the idea to go shopping or see the doctor by bicycle as long as they are inspired by others who do the same. This analysis indicates that both shopping and other trips 
have the highest potential for increasing their bike mode share following if a city’s biking culture improves.

In reality, however, better infrastructure for bicycles and improved biking culture complement, rather than supplement each other. Of course, if a new bicycle facility increases the bike modal split, this higher bike mode share will, in return, have a positive impact on social network effects. At the same time, municipalities with a strong biking culture are more inclined to invest in bicycle infrastructure. Hence, there may be some truth to the claim that not only biking culture is endogenous to bicycle infrastructure, but also vice versa.

On the other hand, while better bicycle facilities will decrease the cost of biking, the lower cost may not necessarily prompt people to start riding the bike, as long as nobody did before. This is the deeper insight of the model results: If people already use their bike, infrastructure improvements will carry a double dividend, one from the lower biking cost and one through the social network effects. If nobody did ride the bicycle before the infrastructure improvement, however, it may very well be possible that the better biking facility will not increase bike ridership. Therefore, in the later case, the focus of bicycle policy should be foremost on improving the city's biking culture. 


\section{CONCLUSION}

This research adds a new dimension to the bike policy discussion by finding that the quantity and quality of bicycle infrastructure is not the only policy variable determining a city's bike mode share, but that in this context biking culture, or social network effects also needs to be considered. Whether or not focusing on improving biking culture turns out to be more cost-effective than building better bicycle facilities, however, remains to be researched.

The regression results presented in this paper show that municipal biking culture has an important impact on the probability of choosing the bike as a transportation mode. To quantify the importance of these social network effects, I additionally ran a regression with the whole dataset (no trip purpose excluded) using just a city-specific dummy variables to capture infrastructure, topography and social network effects combined. Then I regressed both infrastructure (bike-lanes-per-capita) and topography together on the cityspecific dummy variables, and finally added in another regression a newly derived social network effects measure (the city’s bike mode share for all trips). The result was that the first two variables account for approximately $40 \%$ of the variation, while social network effects account for another $45 \%$ (see Table 4.6). 


\section{Table 4.6 about here}

This alternative approach confirms that biking culture, controlled for infrastructure and topography, has explanatory power. If the social network effects instrument would not be included, it is expected that an omitted variable bias will develop, making the model inconsistent. The bias will then most likely be captured in the infrastructure variable, which is strongly correlated with social network effects.

The result that social network effects are important is also visible in the city-specific dummy coefficient estimate for Münster, which was not significantly different from zero even at the ten-percent level. The interpretation of the result would be that in Münster nothing deters people from riding the bike. As the average person is indifferent between using the bicycle and all the other transportation modes, the bike must be viewed as essentially equal to any other transportation mode. On the other hand, the dummy regression coefficients of all the other cities have a negative sign and are significantly different from zero at the one-percent level. This result shows that Münster, which is considered the biking capital of Germany and where one third of all trips are done by bicycle, has indeed a stronger biking culture than the other cities included in the study. 
Bike mode choice and biking culture may be a simultaneous process, possibly making the social network effects variable endogenous. The consequence would be that the coefficient estimate for social network effects is upwardly biased. My approach to circumvent this problem was to use an instrument derived from the excluded trip purpose records.

Another simultaneous process could be biking culture and infrastructure, which would explain the correlation between both variables. This means that the model may exhibit some level of multicollinearity. The coefficient estimates of all other variables would be still unbiased, but the model as a whole would become less robust. However, except for model (2), all the regression coefficients in the models are stable.

All together, this paper presented an innovative approach to analyze bicycle mode share differences in German municipalities, which could change the way bike policy is conducted in the future. The results of the models emphasize the importance of the city's biking culture as a determinate of an individual's probability to choose the bike as the mode of transportation.

\section{REFERENCES}

ADFC-Allgemeiner Deutscher Fahrrad Club (2002) Fahrradklimatest 2005. Bremen, Germany. 
BMVBW-Bundesministerium für Verkehrs, Bau- und Wohnungswesen (2003) Mobility in Germany 2002. Bonn, Germany.

Brock, W. and S. Durlauf (2001) Discrete Choice with Social Interactions, Review of Economic Studies, 68, pp.235-260.

Brock, W. and S. Durlauf (2002) A Multinomial-Choice Model of Neighborhood Effects, American Economic Review, 92, pp. 298-303.

Dugundji, E.R. and J.L. Walker (2005) Discrete Choice with Social and Spatial Network Interdependencies. An Empirical Example Using Mixed GEV Models with Field and "Panel” Effects, Transportation Research Record, forthcoming.

Flade A., Lohmann G., Hacke U., Borcherding K. and W. Bohle (2002) Einflussgrößen und Motive der Fahrradnutzung im Alltagsverkehr. Darmstadt, Germany: Institut Wohnen und Umwelt.

Goetzke, F. (2006a) Mode Choice and Social Networks: The Economics of Walking, Bicycling and Public Transit Use. Morgantown, WV: West Virginia University (dissertation essay). 
Goetzke, F. (2006b) Social Network Effects in Public Transit Use: Evidence from a Spatially Autoregressive Mode Choice Model, Urban Studies, forthcoming.

McFadden, D. (1974) Conditional Logit Analysis of Qualitative Choice Behavior, in: P. Zarembka (ed.) Frontiers in Econometrics. New York, NY: Academic Press, pp. 105-162.

Nelson, A. and D. Allen (1997) If You Build Them, Commuters Will Use Them: Association between Bicycle Facilities and Bicycle Commuting, Transportation Research Record, 1578, pp. 79-83.

Ortuzar, J., Iacobelli, A. and C. Valeze (2000) Estimating Demand for a CycleWay Network, Transportation Research Part A, 34, pp. 353-373.

Páez A. and D. M. Scott (2005) A Discrete Choice Approach to Modeling Social Influence on Individual Decision Making. Seattle, WA: 51st Annual North American Meetings of the Regional Science Association International (revised version of the presentation).

Páez A. and D. M. Scott (2006) Social Influence on Travel Behavior: A Simulation Example of the Decision to Telecommute, Environment and Planning $A$, forthcoming. 
Pez P. (1998) Verkehrsmittelwahl im Stadtbereich und ihre Beeinflußbarkeit. Eine verkehrsgeographische Analyse am Beispiel von Kiel und Lüneburg. Kiel, Germany: Geography Department, University of Kiel.

Rave T. (2005) Bicycle Commuting, in: Arnott R., Rave T. and R. Schöb (eds.), Alleviating Urban Traffic Congestion, Cambridge, MA: MIT Press, 101-134.

Rietveld P. and D. Daniel (2004) Determinants of Bicycle Use: Do Municipal Policies Matter? Transportation Research Part A, 38, pp. 531-550.

Shy O. (2001) Economics of Network Industries. Cambridge, MA and New York, NY: Cambridge University Press.

Siu Y.L., Wardman, M.R.; Page, M. and M.R. Tight (2000) Propensity to Consider Cycle for Commuting Trips. Leeds, UK: Working Paper 544, Institute for Transport Studies, University of Leeds. 
Table 4.1: Summary statistics for included model variables.

\begin{tabular}{|c|c|c|c|c|c|c|}
\hline & Source & $\mathbf{N}$ & Min. & Max. & Mean & S.E. \\
\hline Bike Trips & MiG 2002 & 8,725 & 0 & 1 & 0.12 & 0.003 \\
\hline $\begin{array}{r}\text { Bike Share (Work \& } \\
\text { Education) }\end{array}$ & MiG 2002 & 8,725 & 0 & 0.41 & 0.15 & 0.001 \\
\hline Bike Share (Shopping) & MiG 2002 & 8,725 & 0 & 0.42 & 0.11 & 0.001 \\
\hline Bike Share (Leisure) & MiG 2002 & 8,725 & 0 & 0.34 & 0.12 & 0.001 \\
\hline Bike Share (Other Trips) & MiG 2002 & 8,725 & 0 & 0.34 & 0.11 & 0.001 \\
\hline Bike Lanes per Capita & $\begin{array}{l}\text { Phone } \\
\text { Survey }\end{array}$ & 6,634 & 0.26 & 1.16 & 0.69 & 0.003 \\
\hline $\begin{array}{r}\text { Bike Infrastructure } \\
\text { Score }\end{array}$ & ADFC & 7,834 & 1.13 & 3.67 & 2.39 & 0.008 \\
\hline Flat Topography & $\begin{array}{c}\text { Own } \\
\text { derivation }\end{array}$ & 8,725 & 0 & 1 & 0.77 & 0.005 \\
\hline Trip Length & MiG 2002 & 8,725 & 0 & 96 & 6.70 & 0.111 \\
\hline Bad Weather & MiG 2002 & 8,725 & 0 & 1 & 0.21 & 0.004 \\
\hline Work Trips & MiG 2002 & 8,725 & 0 & 1 & 0.22 & 0.004 \\
\hline Educational Trips & MiG 2002 & 8,725 & 0 & 1 & 0.04 & 0.002 \\
\hline Shopping Trips & MiG 2002 & 8,725 & 0 & 1 & 0.29 & 0.005 \\
\hline Leisure Trips & MiG 2002 & 8,725 & 0 & 1 & 0.28 & 0.005 \\
\hline Other Trips & MiG 2002 & 8,725 & 0 & 1 & 0.18 & 0.004 \\
\hline Male & MiG 2002 & 8,725 & 0 & 1 & 0.45 & 0.005 \\
\hline Age & MiG 2002 & 8,725 & 15 & 91 & 46.45 & 0.184 \\
\hline Poor & MiG 2002 & 8,725 & 0 & 1 & 0.14 & 0.004 \\
\hline
\end{tabular}


Table 4.2: Municipal characteristics used in the model.

\begin{tabular}{|c|c|c|c|c|}
\hline Municipality & $\begin{array}{c}\text { Flat } \\
\text { Topography }\end{array}$ & $\begin{array}{l}\text { Total Bike } \\
\text { Share }\end{array}$ & $\begin{array}{c}\text { Bike Lane per } \\
\text { Capita* }\end{array}$ & $\begin{array}{c}\text { Bike Infra- } \\
\text { structure Score }\end{array}$ \\
\hline Aachen & 1 & $10.3 \%$ & 0.47 & \\
\hline Arnsberg & 0 & $7.6 \%$ & 0.64 & \\
\hline Bielefeld & 1 & $6.9 \%$ & 1.10 & 2.31 \\
\hline Bonn & 1 & $12.2 \%$ & 0.80 & 2.59 \\
\hline Darmstadt & 1 & $19.1 \%$ & 1.16 & 2.07 \\
\hline Delmenhorst & 1 & $21.0 \%$ & & 2.52 \\
\hline Düsseldorf & 1 & $18.4 \%$ & 0.61 & 1.15 \\
\hline Frankfurt & 1 & $9.5 \%$ & & 1.76 \\
\hline Hamm & 1 & $8.5 \%$ & 0.87 & 2.64 \\
\hline Hanau & 1 & $8.0 \%$ & & 1.70 \\
\hline Hannover & 1 & $14.3 \%$ & 0.71 & 2.94 \\
\hline Kassel & 0 & $1.0 \%$ & 0.35 & 1.34 \\
\hline Köln & 1 & $9.9 \%$ & & 1.60 \\
\hline Marburg & 0 & $9.4 \%$ & 1.03 & \\
\hline Münster & 1 & $35.4 \%$ & 0.98 & 3.67 \\
\hline Neuss & 1 & $6.2 \%$ & 0.50 & 2.28 \\
\hline Offenbach & 1 & $3.8 \%$ & 0.64 & \\
\hline Oldenburg & 1 & $25.3 \%$ & 0.94 & 3.16 \\
\hline Recklinghausen & 1 & $2.8 \%$ & 0.79 & \\
\hline Rüsselsheim & 1 & $11.8 \%$ & 1.02 & 2.78 \\
\hline Velbert & 0 & $4.2 \%$ & 1.14 & \\
\hline Viersen & 1 & $11.0 \%$ & 0.94 & \\
\hline Wesel & 1 & $16.8 \%$ & 0.94 & 3.42 \\
\hline Wiesbaden & 0 & $2.4 \%$ & 0.26 & 0.72 \\
\hline
\end{tabular}

* in km per 1,000 capita 
Table 4.3: Coefficients for the correlations of the network proxies with total bike model share and the two different infrastructure variables.

\begin{tabular}{rccc}
\hline $\begin{array}{c}\text { Network Proxies (Based on } \\
\text { the Bike Mode Share of } \\
\text { Excluded Trip Purpose) }\end{array}$ & $\begin{array}{c}\text { Total Bike } \\
\text { Mode Share }\end{array}$ & $\begin{array}{c}\text { Bike Lane } \\
\text { per Capita }\end{array}$ & $\begin{array}{c}\text { Bike Infra- } \\
\text { structure } \\
\text { Score }\end{array}$ \\
\hline Work and Education & $0.901^{* * *}$ & 0.295 & $0.782^{* * *}$ \\
Shopping & $0.927^{* * *}$ & $0.407^{*}$ & $0.541^{* *}$ \\
Leisure & $0.872^{* * *}$ & 0.335 & $0.475^{*}$ \\
Other & $0.789^{* * *}$ & $0.426^{*}$ & $0.421^{*}$ \\
\hline
\end{tabular}

*** significant at the 1-percent level

** significant at the 5-percent level

* $\quad$ significant at the 10 -percent level 
Table 4.4(a): Binary logistic regression results for bicycle trips using "Bike Lanes per Capita" as the infrastructure measure. The network effect proxy is the bike mode share of the excluded trip purpose.

\begin{tabular}{|c|c|c|c|c|}
\hline & (1) & (2) & (3) & (4) \\
\hline \multirow[b]{3}{*}{ Bike Lanes per Capita } & $\begin{array}{c}-3.708 * * * \\
(0.256)\end{array}$ & $\begin{array}{c}-3.218 * * * \\
(0.256)\end{array}$ & $\begin{array}{c}-3.960 * * * \\
(0.316)\end{array}$ & $\begin{array}{c}-3.250 * * * \\
(0.245)\end{array}$ \\
\hline & $\begin{array}{c}5.248 * * * \\
(0.703)\end{array}$ & $\begin{array}{c}4.279 * * * \\
(0.662)\end{array}$ & $\begin{array}{c}6.112^{* * *} \\
(0.805)\end{array}$ & $\begin{array}{c}3.126 * * * \\
(0.560)\end{array}$ \\
\hline & $\begin{array}{c}1.028 * * * \\
(0.268)\end{array}$ & $\begin{array}{l}0.525^{*} \\
(0.280)\end{array}$ & $\begin{array}{c}0.814^{* * *} \\
(0.283)\end{array}$ & $\begin{array}{l}0.492 * \\
(0.268)\end{array}$ \\
\hline Flat Topography & $\begin{array}{c}0.423 * * \\
(0.192)\end{array}$ & $\begin{array}{c}0.665^{* * *} \\
(0.178)\end{array}$ & $\begin{array}{c}1.318^{* * *} \\
(0.198)\end{array}$ & $\begin{array}{c}1.011^{* * *} \\
(0.168)\end{array}$ \\
\hline Trip Length & $\begin{array}{c}-0.052 * * * \\
(0.010)\end{array}$ & $\begin{array}{c}-0.084 * * * \\
(0.010)\end{array}$ & $\begin{array}{c}-0.196 * * * \\
(0.017)\end{array}$ & $\begin{array}{c}-0.093 * * * \\
(0.010)\end{array}$ \\
\hline Bad Weather & $\begin{array}{c}-0.467 * * * \\
(0.125)\end{array}$ & $\begin{array}{c}-0.364 * * * \\
(0.122)\end{array}$ & $\begin{array}{c}-0.443 * * * \\
(0.126)\end{array}$ & $\begin{array}{c}-0.417 * * * \\
(0.113)\end{array}$ \\
\hline Work Trips & Excluded & $\begin{array}{c}0.306^{* * *} \\
(0.113)\end{array}$ & $\begin{array}{c}0.748 * * * \\
(0.138)\end{array}$ & $\begin{array}{c}0.316^{* * *} \\
(0.112)\end{array}$ \\
\hline Educational Trips & Excluded & $\begin{array}{c}0.672 * * * \\
(0.190)\end{array}$ & $\begin{array}{c}1.027 * * * \\
(0.216)\end{array}$ & $\begin{array}{c}0.682 * * * \\
(0.188)\end{array}$ \\
\hline Shopping Trips & $\begin{array}{l}-0.152 \\
(0.108)\end{array}$ & Excluded & $\begin{array}{l}-0.012 \\
(0.127)\end{array}$ & $\begin{array}{l}-0.207 * \\
(0.107)\end{array}$ \\
\hline Leisure Trips & Base & Base & Excluded & Base \\
\hline Other Trips & $\begin{array}{c}-0.243 * \\
(0.125)\end{array}$ & $\begin{array}{c}-0.242 * \\
(0.125)\end{array}$ & Base & Excluded \\
\hline Male & $\begin{array}{c}0.268 * * * \\
(0.094)\end{array}$ & $\begin{array}{c}0.402^{* * *} \\
(0.093)\end{array}$ & $\begin{array}{c}0.264^{* * *} \\
(0.097)\end{array}$ & $\begin{array}{c}0.266 * * * \\
(0.086)\end{array}$ \\
\hline Age & $\begin{array}{l}-0.001 \\
(0.003)\end{array}$ & $\begin{array}{c}-0.001 \\
(0.003)\end{array}$ & $\begin{array}{c}-0.004 \\
(0.003)\end{array}$ & $\begin{array}{c}0.000 \\
(0.003)\end{array}$ \\
\hline Poor & $\begin{array}{c}0.320 * * \\
(0.126)\end{array}$ & $\begin{array}{c}0.207 \\
(0.128) \\
\end{array}$ & $\begin{array}{c}0.017 \\
(0.133) \\
\end{array}$ & $\begin{array}{c}0.237 * * \\
(0.118)\end{array}$ \\
\hline Sample Size & 4,927 & 4,739 & 4,779 & 5,457 \\
\hline - 2 Log-Likelihood & $3,180.09$ & 3,175.93 & $2,944.73$ & $3,686.91$ \\
\hline
\end{tabular}


Table 4.4(b): Binary logistic regression results for bicycle trips using "Bike Infrastructure Score" as the infrastructure measure. The network effect proxy is the bike mode share of the excluded trip purpose.

\begin{tabular}{|c|c|c|c|c|}
\hline & (5) & (6) & (7) & (8) \\
\hline \multirow{4}{*}{$\begin{array}{l}\text { Bike Infrastructure } \\
\text { Score } \\
\qquad \text { Flat Topography }\end{array}$} & $\begin{array}{c}-3.349 * * * \\
(0.261)\end{array}$ & $\begin{array}{c}-3.440 * * * \\
(0.259)\end{array}$ & $\begin{array}{c}-3.875^{* * *} \\
(0.311)\end{array}$ & $\begin{array}{c}-3.504 * * * \\
(0.251)\end{array}$ \\
\hline & $\begin{array}{c}6.690 * * * \\
(0.782)\end{array}$ & $\begin{array}{c}3.893 * * * \\
(0.661)\end{array}$ & $\begin{array}{c}5.877^{* * *} \\
(0.743)\end{array}$ & $\begin{array}{c}3.136 * * * \\
(0.470)\end{array}$ \\
\hline & $\begin{array}{c}-0.261^{* * *} \\
(0.096)\end{array}$ & $\begin{array}{c}0.249 * * * \\
(0.079)\end{array}$ & $\begin{array}{c}0.262 * * * \\
(0.075)\end{array}$ & $\begin{array}{c}0.306^{* * *} \\
(0.070)\end{array}$ \\
\hline & $\begin{array}{c}1.1529 * * * \\
(0.253)\end{array}$ & $\begin{array}{c}0.926 * * * \\
(0.251)\end{array}$ & $\begin{array}{c}1.198 * * * \\
(0.292)\end{array}$ & $\begin{array}{c}1.072 * * * \\
(0.242)\end{array}$ \\
\hline Trip Length & $\begin{array}{c}-0.061^{* * *} \\
(0.009)\end{array}$ & $\begin{array}{c}-0.087^{* * *} \\
(0.009)\end{array}$ & $\begin{array}{c}-0.190 * * * \\
(0.015)\end{array}$ & $\begin{array}{c}-0.099 * * * \\
(0.010)\end{array}$ \\
\hline Bad Weather & $\begin{array}{c}-0.610 * * * \\
(0.116)\end{array}$ & $\begin{array}{c}-0.534 * * * \\
(0.115)\end{array}$ & $\begin{array}{c}-0.520 * * * \\
(0.115)\end{array}$ & $\begin{array}{c}-0.528 * * * \\
(0.106)\end{array}$ \\
\hline Work Trips & Excluded & $\begin{array}{c}0.218^{* *} \\
(0.103)\end{array}$ & $\begin{array}{c}0.643^{* * *} \\
(0.123)\end{array}$ & $\begin{array}{c}0.247^{* *} \\
(0.103)\end{array}$ \\
\hline Educational Trips & Excluded & $\begin{array}{c}0.542 * * * \\
(0.176)\end{array}$ & $\begin{array}{c}0.933 * * * \\
(0.197)\end{array}$ & $\begin{array}{c}0.573 * * * \\
(0.175)\end{array}$ \\
\hline Shopping Trips & $\begin{array}{l}-0.153 \\
(0.096)\end{array}$ & Excluded & $\begin{array}{c}-0.033 \\
(0.111)\end{array}$ & $\begin{array}{c}-0.188 * * \\
(0.096)\end{array}$ \\
\hline Leisure Trips & Base & Base & Excluded & Base \\
\hline Other Trips & $\begin{array}{l}-0.184 * \\
(0.110)\end{array}$ & $\begin{array}{l}-0.177 \\
(0.110)\end{array}$ & Base & Excluded \\
\hline Male & $\begin{array}{c}0.208 * * \\
(0.084)\end{array}$ & $\begin{array}{c}0.376^{* * * *} \\
(0.084)\end{array}$ & $\begin{array}{c}0.254 * * * \\
(0.086)\end{array}$ & $\begin{array}{c}0.210^{* * *} \\
(0.078)\end{array}$ \\
\hline Age & $\begin{array}{c}-0.002 \\
(0.002)\end{array}$ & $\begin{array}{c}-0.003 \\
(0.003)\end{array}$ & $\begin{array}{c}-0.003 \\
(0.003)\end{array}$ & $\begin{array}{c}-0.002 \\
(0.002)\end{array}$ \\
\hline Poor & $\begin{array}{c}0.103 \\
(0.115) \\
\end{array}$ & $\begin{array}{c}0.058 \\
(0.120) \\
\end{array}$ & $\begin{array}{c}-0.157 \\
(0.122) \\
\end{array}$ & $\begin{array}{c}0.037 \\
(0.110) \\
\end{array}$ \\
\hline Sample Size & 5,819 & 5,564 & 5,675 & 6,444 \\
\hline - 2 Log-Likelihood & 3,981.92 & 3,827.32 & $3,678.07$ & $4,455.02$ \\
\hline
\end{tabular}


Table 4.5(a): Marginal effects for regressions (1) through (4).

\begin{tabular}{|c|c|c|c|c|}
\hline & (1) & (2) & (3) & (4) \\
\hline Network Effects & 0.5252 & 0.4622 & 0.6468 & 0.3393 \\
\hline Bike Lanes per Capita & 0.1029 & 0.0567 & 0.0861 & 0.0534 \\
\hline Flat Topography & 0.0423 & 0.0718 & 0.1395 & 0.1097 \\
\hline Trip Length & -0.0052 & -0.0091 & -0.0207 & -0.0101 \\
\hline Bad Weather & -0.0467 & -0.0393 & -0.0469 & -0.0453 \\
\hline Work Trips & Excluded & 0.0331 & 0.0792 & 0.0343 \\
\hline Educational Trips & Excluded & 0.0726 & 0.1087 & 0.0740 \\
\hline Shopping Trips & -0.0152 & Excluded & -0.0013 & -0.0225 \\
\hline Leisure Trips & Base & Base & Excluded & Base \\
\hline Other Trips & -0.0243 & -0.0261 & Base & Excluded \\
\hline Male & 0.0268 & 0.0434 & 0.0279 & 0.0289 \\
\hline Age & -0.0001 & -0.0001 & -0.0004 & 0.0000 \\
\hline Poor & 0.0320 & 0.0224 & 0.0018 & 0.0257 \\
\hline
\end{tabular}


Table 4.5(b): Marginal effects for regressions (5) through (8).

\begin{tabular}{|c|c|c|c|c|}
\hline & (5) & (6) & (7) & (8) \\
\hline Network Effects & 0.7201 & 0.4348 & 0.6494 & 0.3524 \\
\hline \multicolumn{5}{|l|}{ Bike Infrastructure } \\
\hline Score & -0.0281 & 0.0278 & 0.0290 & 0.0344 \\
\hline Flat Topography & 0.1241 & 0.1034 & 0.1324 & 0.1204 \\
\hline Trip Length & -0.0066 & -0.0097 & -0.0210 & -0.0111 \\
\hline Bad Weather & -0.0657 & -0.0596 & -0.0575 & -0.0593 \\
\hline Work Trips & Excluded & 0.0243 & 0.0711 & 0.0278 \\
\hline Educational Trips & Excluded & 0.0605 & 0.1031 & 0.0644 \\
\hline Shopping Trips & -0.0165 & Excluded & -0.0036 & -0.0211 \\
\hline Leisure Trips & Base & Base & Excluded & Base \\
\hline Other Trips & -0.0198 & -0.0198 & Base & Excluded \\
\hline Male & 0.0224 & 0.0420 & 0.0281 & 0.0236 \\
\hline Age & -0.0002 & -0.0003 & -0.0003 & -0.0002 \\
\hline Poor & 0.0111 & 0.0065 & -0.0173 & 0.0042 \\
\hline
\end{tabular}


Table 4.6: Regression results for city-specific dummies as the dependent variable.

\begin{tabular}{|c|c|c|}
\hline & (1) & (2) \\
\hline Constant Term & $\begin{array}{c}-3.947 * * * \\
(0.296)\end{array}$ & $\begin{array}{c}-3.784 * * * \\
(0.296)\end{array}$ \\
\hline Network Effects & & $\begin{array}{c}8.497 * * * \\
(1.138)\end{array}$ \\
\hline Bike Lanes per Capita & $\begin{array}{c}1.456^{* *} \\
(0.652)\end{array}$ & $\begin{array}{c}0.607 * \\
(0.337)\end{array}$ \\
\hline Flat Topography & $\begin{array}{c}0.992 * * \\
(0.387) \\
\end{array}$ & $\begin{array}{l}0.387 * \\
(0.205) \\
\end{array}$ \\
\hline Sample size & 17 & 17 \\
\hline Adjusted $\mathbf{R}^{2}$ & 0.41 & 0.86 \\
\hline
\end{tabular}


Chapter 5

Conclusion 
While transportation planners were always aware of social network effects in transportation mode choice modeling, recently they have gained more interest for academic researchers. My three dissertation essays give further insight in both the theoretical foundation as well as the empirical analysis of social network effects in mode choice modeling.

In my first, more analytically oriented essay, I start out by developing a mode choice model with just one transportation mode, which I later extend to include a second, alternative mode. I describe social network effects as a coordination externality with the potential problem of having a Nash equilibrium that is not welfare maximizing. In this context I shed new light on the collapse of transit ridership in the United States after World War II.

The other two essays are econometric applications of social network effects. In the second essay, I investigate social network effects in transit ridership using travel behavior data from New York City. In the third essay, I explain bike mode share differences in German cities through social network effects. In both cases I had to deal with the problem of social network effects being endogenous. In the New York dataset, I resolved the issue by choosing to use a spatially autoregressive process for social network effects. For the German dataset, I decided to derive an instrument from records with excluded 
trip purposes. Both models empirically indicate the existence of social network effects for transit use in New York and bicycling in Germany, respectively.

The main contribution of the dissertation, however, is to emphasize the importance of social network effects for transportation policy. This issue runs through all three essays like a red thread. In the theoretical essay, I establish the possibility that there exist multiple mode share equilibria, and that a city's mode share is path-dependent. Hence, certain unique historical events may result in irreversible changes to transportation mode shares. Then, in the second essay, I show that ignoring social network effects may lead to an omitted variable bias, with the result of systematically overestimating suburban transit ridership, and underestimating transit ridership in the CBD. Finally, in my third essay, I contrast biking policies relying on infrastructure improvement with a policy approach that considers strengthening the city's biking culture.

At the end of a research project, one always recognizes what could have been done better. This, of course, applies to my dissertation as well, which I interpret as a sign of successful learning. The two issues in need of further investigation would be the endogeneity of social network effects and the self-selection of residential location choice. A possible improvement for the first issue of endogeneity may be a simultaneous equation approach with a 
linear probability model formulation, while a nested logit choice model could help to alleviate the second issue of self-selection.

I see this dissertation as the beginning of my research agenda, in laying the groundwork for the topic of social network effects in transportation mode choice modeling, which I will build upon. I plan to improve the modeling approaches by addressing the endogeneity and self-selection issues in future papers.

Another problem in these models is that residential location choice depends on transportation infrastructure. To complicate the issue, residential location choice, again, will depend on social network effects. While the Tiebout model does not explicitly include social network effects in such a way as it is modeled in my dissertation, voting by feet is an expression of social network effects. Hence, based on the methodological fundaments laid in my dissertation, it would be a challenging but also exciting exercise for me to improve present-day land-use and residential choice model by treating transportation infrastructure as endogenous and accounting for social network effects, as well.

Two similar fields of social network effects applications in urban and regional economics are immigration and fertility decision making. The two research areas are especially interesting for me as a German, coming from the 
country with the lowest reproduction rate in the world and, therefore, more than ever dependent on immigration. Both these issues are inherently interlinked: It is expected that with the depopulation of German cities, some cities are in danger of collapse, while other cities may even continue to grow, mainly do to interregional as well as international immigration. As a sideline, I should mention that the cities currently growing also have a highest reproduction rate (and not rural areas).

I expect that the concept of social network effects with its many applications will become more important in future policy analysis. The revelation of the dynamic character inherent to social network effects will deepen the insight of any policy issue to be analyzed, and therefore strengthen the derived recommendations. Better access to improved modeling techniques will make empirical estimations of social network effects more operational for planners and consultants. 